\title{
Cellulose Nanocrystals from Native and Mercerized Cotton
}

\section{Somia Haouache}

INRAE Île-de-France-Versailles-Grignon: Institut National de Recherche pour I'Agriculture I'Alimentation et I'Environnement Centre Ile-de-France-Versailles-Grignon

\section{Clara Jimenez-Saelices}

INRAE Pays de la Loire: Institut National de Recherche pour l'Agriculture l'Alimentation et l'Environnement Centre Pays de la Loire

\section{fabrice cousin}

LLB: Laboratoire Leon Brillouin

\section{Xavier Falourd}

INRAE Pays de la Loire: Institut National de Recherche pour l'Agriculture l'Alimentation et l'Environnement Centre Pays de la Loire

\section{bruno pontoire}

INRAE Pays de la Loire: Institut National de Recherche pour l'Agriculture l'Alimentation et l'Environnement Centre Pays de la Loire

\section{karine cahier}

INRAE Pays de la Loire: Institut National de Recherche pour l'Agriculture l'Alimentation et I'Environnement Centre Pays de la Loire

\section{francois jerome}

Poitiers University: Universite de Poitiers

\section{Isabelle Capron ( $\boldsymbol{Q}$ isabelle.capron@inrae.fr)}

INRAE Pays de la Loire: Institut National de Recherche pour l'Agriculture l'Alimentation et l'Environnement Centre Pays de la Loire

\section{Research Article}

Keywords: cellulose nanocrystals, mercerization, cellulose II, biobased nanoparticles, nanostructuration.

Posted Date: April 30th, 2021

DOI: https://doi.org/10.21203/rs.3.rs-415820/v1

License: (c) (1) This work is licensed under a Creative Commons Attribution 4.0 International License. Read Full License 
Version of Record: A version of this preprint was published at Cellulose on January 24th, 2022. See the published version at https://doi.org/10.1007/s10570-021-04313-8. 


\section{Cellulose Nanocrystals from native and 2 mercerized cotton}

3 Somia Haouache ${ }^{1,2}$, Clara Jimenez-Saelices ${ }^{1}$, Fabrice Cousin ${ }^{3}$, Xavier Falourd ${ }^{1}$, Bruno

4 Pontoire $^{1}$, Karine Cahier ${ }^{1}$, François Jérome ${ }^{2}$ and Isabelle Capron ${ }^{1^{*}}$

$5 \quad{ }^{1}$ UR1268 Biopolymères Interactions Assemblages, INRAE, 44316 Nantes, France

${ }^{2}$ Institut de Chimie des Milieux et Matériaux de Poitiers, Université de Poitiers, 86073

7 Poitiers, France

$8{ }^{3}$ Laboratoire Léon Brillouin, Université Paris-Saclay, CEA-Saclay, Gif-sur-Yvette, France

*Correspondence: isabelle.capron@inrae.fr; Tel.: +33-24067-5095; orcid: 0000-0001-9145-3803

Abstract: Nanocelluloses occur under various crystalline forms that are being selectively used for mercerized by alkaline treatment (CF-II) without molar mass variation $(560000 \mathrm{~g} / \mathrm{mol})$ and both were acid hydrolyzed, forming cellulose nanocrystals in native (CNC-I) and mercerized (CNC-II) forms. This work establishes detailed characterization of these two nanoparticles morphology (light and neutron scattering, TEM, AFM), surface chemistry (zetametry and surface charge), crystallinity (XRD, ${ }^{13} \mathrm{C} N M R$ ), and average molar mass coupled to chromatographic technics (SECMALLS-RI, A4F-MALLS-RI), evidencing variations in packing of the crystalline domains. The crystal size of CNC-II is reduced by half compared to CNC-I, with molar masses of individual chains of 41 $000 \mathrm{~g} / \mathrm{mol}$ and $22000 \mathrm{~g} / \mathrm{mol}$ for CNC-I and CNC-II respectively, whereas the same charged surface chemistry is measured. This fundamental analysis may give insight to new applicative development.

Keywords: cellulose nanocrystals, mercerization, cellulose II, biobased nanoparticles, nanostructuration.

\section{Introduction}

Cellulose is a linear homopolysaccharide of D-glucopyranose units connected by $\beta(1-4)$ glycosidic bonds (Habibi, Lucia, and Rojas 2010; Moon et al. 2011; Nishiyama 2009)(Nishiyama 2009)(Nishiyama 2009) stabilized by an inter and intramolecular complex network of hydrogen bonds and van der Waals interactions. According to the association type, cellulose exists in six crystalline forms called cellulose I, II, III-I, III-II, IV-I and IV-II (Kroon-Batenburg, Bouma, and Kroon 1996). Cellulose I correspond to fibrillary native cellulose with parallel oriented chains. The other forms are obtained by conversion of type I by chemical and/or thermal treatments (Gardner and Blackwell 1974; Atalla and VanderHart 1999; Nishiyama, Langan et al. 2002). Cellulose I can undergo an irreversible transition into a more thermodynamically stable crystalline form that is cellulose II by two distinct processes; regeneration or mercerization. Mercerization involves intracrystalline swelling of the cellulose in concentrated aqueous $\mathrm{NaOH}$ where the limit concentration depends on the temperature between 8-15\%, with lower temperature allowing transformation at lower concentration (Warwicker 1967; Duchemin 2015) where chains change their orientation from original parallel chains of cellulose I to antiparallel chains (opposite polarity) 
(Kolpak, Weih, and Blackwell 1978; Stipanovic and Sarko 1976; Fink and Philipp 1985). The mechanism of mercerization has long been studied. An interdigitation mechanism was first proposed by Okano and Sarko (Okano and Sarko 1985)(Okano and Sarko 1985)(Okano and Sarko 1985)(Okano and Sarko 1985). $\mathrm{NaOH}$ is absorbed converting cellulose I into a swollen structure in which all contacts between adjacent chains are removed. Once $\mathrm{NaOH}$ has been removed by washing with water, a bi-oriented structure cellulose II is obtained (Paul Langan, Nishiyama, and Chanzy 2001)(Langan, Nishiyama et al. 1999; Langan, Nishiyama et al. 2001)(Langan, Nishiyama et al. 1999; Langan, Nishiyama et al. 2001)(Langan, Nishiyama et al. 1999; Langan, Nishiyama et al. 2001).

Nishiyama et al. (Nishiyama, Kuga, and Okano 2000) proposed a molecular association in Na-Cellulose where van der Waals' interaction is the driving force of the formation of cellulose II. The effect of mercerization on the crystallinity was investigated for different native cellulose samples (J. F. Revol, Dietrich, and Goring 1987)(Revol, Dietrich et al. 1987)(Revol, Dietrich et al. 1987)(Revol, Dietrich et al. 1987), all cellulose II obtained have a narrow range of crystallinity and a substantially constant crystal size, whereas non-native celluloses treated have a wide range of crystallinity and crystal size. In this study, it was also shown that in the case of highly crystalline cellulose, mercerization reduces crystallinity and crystal size, whereas in the case of low crystallinity cellulose, mercerization increases crystallinity and the size of the crystal. These trends would not be expected if the conversion of cellulose I to cellulose II was simply a change in conformation of the chain or arrangement of atoms. These results are more in line with the idea that mercerization involves a complete destruction of the structure of cellulose I by separation of the molecular chains followed by reforming of the crystalline structure in the form of cellulose II. These results are consistent with the hypothesis that mercerization involves a mixture of adjacent and antiparallel cellulose microfibers (Okano and Sarko 1985).

Mercerized cellulose nanocrystals can be obtained from acid hydrolysis of mercerized fibers (Neto et al. 2016)(Neto, Putaux et al. 2016)(Neto, Putaux et al. 2016)(Neto, Putaux et al. 2016). The nanocrystals (CNC-II) obtained by Sebe et al. were found significantly different from that of the needle-shaped CNC-I: smaller and ribbon shaped with rounded tips while Neto et al. described CNC-II shorter (from $240 \mathrm{~nm}$ to 132 $\mathrm{nm}$ ) broader (from $15 \mathrm{~nm}$ to $19 \mathrm{~nm}$ ) with identical thickness (around $4 \mathrm{~nm}$ ), and with an increased crystallinity from $56 \%$ to $68 \%$. For $\mathrm{Li}$ et al., the mercerized CNCs were even much smaller (19 nm in length and $11 \mathrm{~nm}$ in width) with ellipsoid shapes.(Li et al. 2018).

CNCs are foreseen to have a major impact in the coming years and variability will be a key of this development. Recent reviews show the interest of the selective modification of the reducing end (Tao, Lavoine et al. 2020; Heise, Delepierre et al. 2021) of CNC-I. A growing interest is now focused on CNC-II with the hemiacetal form at the two extremities. Therefore, a precise control of their various forms is of great importance but the transition mechanism is still matter of debate. In the present work, native (CF-I) and mercerized (CF-II) cotton fibers are both hydrolyzed using the same sulfuric acid hydrolysis process leading to CNC-I and CNC-II. A full set of complementary techniques is described and used to precisely characterize the morphology, molar mass, structure, surface charge, and degree of polymerization of both nanocrystals.

\section{Materials and Methods}

Materials: The native cotton cellulose fibers were obtained from Buckeye Technologie Inc, US. All reactants were acquired from Sigma Aldrich and used without further purification. Ultrapure water was produced with the Milli-Q reagent system (18.2 M $\Omega$ $\mathrm{cm}$ Millipore Milli-Q purification system). 
Cellulose samples preparation: Native cotton cellulose fiber (CF-I) was mercerized (CF-II) according to a protocol similar to that described by Neto et al (Neto et al. 2016). $10 \mathrm{~g}$ of CF-I were introduced into $300 \mathrm{~mL}$ of $20 \mathrm{wt} \% \mathrm{NaOH}$ and mechanically stirred for 5 $\mathrm{h}$ at $25^{\circ} \mathrm{C}$. The mixture was washed several times with distilled water in order to remove the $\mathrm{NaOH}$ solution, and then dried at $40{ }^{\circ} \mathrm{C}$ for $48 \mathrm{~h}$. This conversion was carried out with a yield of $100 \%$.

Preparation of cellulose nanocrystals (CNC-I and CNC-II): Both CNCS were prepared by hydrolysis with sulfuric acid according to the method of Revol et al ( Revol et al. 1992) with minor modifications. Briefly, cellulose nanocrystals (CNC-I and CNC-II) were prepared under the same conditions from fibers (CF-I and CF-II) respectively using sulfuric acid hydrolysis at $64 \%$ at $68{ }^{\circ} \mathrm{C}$ under stirring for $20 \mathrm{~min}$. After hydrolysis, the suspensions were washed by centrifugation, dialyzed to neutrality against Milli- $Q$ water for 2 weeks, and deionized using mixed bed resin (TMD-8). The final dispersion was sonicated for $10 \mathrm{~min}$, filtered, and stored at $4{ }^{\circ} \mathrm{C}$. The yield was $64 \%$ and $40 \%$ for CNC-I and CNC-II respectively.

\section{Cellulose samples characterization}

$X$-ray Diffraction. The determination of crystalline type, crystallinity index and crystal size of the different samples was performed by X-ray Diffraction (XRD) analysis using a Bruker D8 Discover diffractometer (Karlsruhe, Germany) equipped with a VANTEC 500 $2 \mathrm{D}$ detector. $X$-ray radiation, CuK $\alpha 1(\lambda=0.15406 \mathrm{~nm})$, produced in a sealed tube at 40 $\mathrm{kV}$ and $40 \mathrm{~mA}$, was selected and parallelized using crossed Göbel mirrors and collimated to produce a beam of 300 or $500 \mu \mathrm{m}$ diameter. The suspensions of nanocrystals were freeze-dried then pressed at room temperature to obtain well-dense pellets, while the fibers were used as such. The diffraction patterns were recorded for $10 \mathrm{~min}$ over a range from $3^{\circ}$ to $40^{\circ}(2 \theta)$. The recorded intensity was normalized by the total peak area to eliminate the influence of the thickness variation and the absorption coefficient of the samples. The X-ray crystallinity index (Xcr) was estimated by the crystalline to amorphous areas using Origin (v8.0891) software.

Solid-state NMR CP-MAS. The NMR experiments were carried out on a Avance III-400 $\mathrm{MHz}$ spectrometer (Bruker; France) operating at $100.62 \mathrm{MHz}$ for $13 \mathrm{C}$, equipped with a double-resonance H/X CP-MAS 4-mm probe for CP-MAS (Cross-Polarization Magic Angle Spinning) solid-state experiments. The samples were wetted and spun at $12000 \mathrm{~Hz}$ at room temperature.

CP-MAS spectra were acquired with a contact time of $1.5 \mathrm{~ms}$ and over accumulation of 2048 scans separate of $10 \mathrm{~s}$ as recycling delay. The carbonyl carbon was set to 176.03 ppm through external glycine calibration. NMR spectra deconvolution was performed using PeakFit ${ }^{\circledR}$ (v.4.11) software (Systat Software, Inc., US). Peak chemical shifts were assigned according to (Newman and Davidson 2004; Larsson et al. 1999). The NMR crystallinity index of CF and CNC was calculated according to (Larsson et al. 1999; Zuckerstätter et al. 2013).

Conductometry. The hydrolysis of the cellulose with sulfuric acid makes it possible to obtain a colloidal suspension of the nanometric-sized crystals and have SO3- charges on their surface. The measurement of the quantity of charges on the CNCs surface charge was performed by conductometric titration with a $0.001 \mathrm{M} \mathrm{NaOH}$ solution using a TIM900 titration manager and a CDM230 conductimeter equipped with a CDC749 conductivity cell.

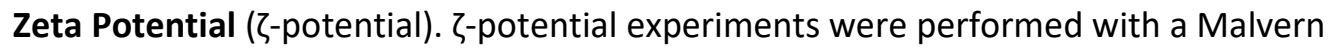
NanoZS instrument. All measurements were made at a temperature of $20^{\circ} \mathrm{C}$ with a 
detection angle of $12.8^{\circ} \mathrm{CNCs}$ dispersions of $1 \mathrm{~g} / \mathrm{L}$ at $\mathrm{pH}=7$ were prepared at $20^{\circ} \mathrm{C}$ and filtered by $5 \mu \mathrm{m}$. Each sample was measured a total of 5 times. The confidence interval (error) presented is the standard deviation of samples measured in triplicate.

\section{Asymmetrical flow field-flow fractionation coupled to Multi-Angle Laser Light} Scattering and Refractive Index (A4F-MALLS-RI) detection. AF4 instrument was coupled with two online detectors: a MALLS instrument (DAWN Heleos II) fitted with a K5 flow cell and a GaAs laser $(\lambda=663 \mathrm{~nm})$, and a refractometric detector operating at the same wavelength (Optilab T-rEX) from Wyatt Technology (Santa Barbara, CA, USA). The AF4 instrument was constituted of an AF4 channel ( $275 \mathrm{~mm}$-long), a spacer $350 \mu \mathrm{m}$ thick and a regenerated cellulose membrane with a nominal cut-off of $10 \mathrm{kDa}$ (Millipore, Bedford, MA, USA). The refractive index increment $\mathrm{dn} / \mathrm{dc}$ was $0.146 \mathrm{~mL} / \mathrm{g}$, value classically used for glucans solubilized in water. The AF4 channel flow, cross flow, sample injection and focus flow were controlled with a Wyatt Eclipse AF4 flow chassis, a pump and an autosampler from ThermoFisher Scientific (Waltham, MA, USA). CNCs dispersions of 0.5 $\mathrm{g} / \mathrm{L}$ in water were prepared at $20^{\circ} \mathrm{C}$ and systematically freshly sonicated (amplitude 5, 8 $\mathrm{s}, 2 \mathrm{on} / 1 \mathrm{off})$ before being injected. Each sample was measured a total of two times. Weight and number average molar masses $\left(\overline{\mathbf{M}}_{w}, \overline{\mathbf{M}}_{n}\right)$ and polydispersity $\left(\overline{\mathbf{M}}_{\mathbf{w}} / \overline{\mathbf{M}}_{\mathbf{n}}\right)$ of CNCs were determined with Wyatt ASTRA ${ }^{\circledR}$ software (v. 6.1.4) with Zimm extrapolation of order 1.

\section{Size Exclusion Chromatography coupled to Multi-Angle Laser Light Scattering and} Refractive Index (SEC-MALLS-RI) detection. The determination of molar mass distribution of chains of cellulose in DMAc/LiCl was carried out at room temperature using OMNISEC SYSTEM Malvern. The size exclusion chromatography (SEC) (OMNISEC Resolve, Malvern) system was coupled with a multi-angle laser light scattering 20 (MALLS) (Malvern) and OMNISEC Reveal devices (Malvern). The SEC columns used were Viscoteck Tguard, LT4000L, LT5000L and LT7000L. The mobile phase used for SEC was $\mathrm{N}, \mathrm{N}$-dimethylacetamide (DMAC) (HPLC grade) containing lithium chloride (LiCl) $0.9 \%$ $(\mathrm{v} / \mathrm{w})$, that had been filtered through $0.6 \mu \mathrm{m}$ polypropylene prefilters. This eluant was chosen because it solubilizes cellulose without significant depolymerization during the dissolution process as well as during storage at room temperature for long periods (Dupont and Harrison 2004; Yanagisawa and Isogai 2005). Calculation of weight and number average molar masses $\left(\overline{\mathbf{M}}_{\mathbf{w}}, \overline{\mathbf{M}}_{\mathbf{n}}\right)$ and polydispersity $\left(\overline{\mathbf{M}}_{\mathbf{w}} / \overline{\mathbf{M}}_{\mathbf{n}}\right)$ of samples were performed with a dn/dc value of $0.136 \mathrm{~mL} / \mathrm{g}$ (Hasani et al. 2013) and determined with OMNISEC software (v.10.30) with Zimm extrapolation of order 2.

Cellulose was solubilized in the DMAc/LiCl 9\% (v/w) (Medronho and Lindman 2015) via solvent exchange steps H2O/Met-OH/DMAc CF-I and CF-II and H2O/Et-OH/DMAc for CNC-I and CNC-II.

For fibers, $100 \mathrm{mg}$ (dry content) of CF-I and CF-II were washed with $30 \mathrm{~mL}$ methanol, the excess of methanol was removed by filtration on fritter $n^{\circ} 3$, and this step was repeated three times. The recovered pellet was washed three times with $30 \mathrm{~mL}$ of DMAc for solvent exchange, the excess of DMAc was removed by filtration on fritter $n^{\circ} 3$. After solvent exchange steps, $10 \mathrm{~mL}$ of DMAc/LiCl $9 \%(\mathrm{v} / \mathrm{w})$ were added to the vial containing the sample and allowed to stir magnetically at $4{ }^{\circ} \mathrm{C}$ for dissolution.

For CNCs, the samples in the form of aqueous suspensions have been freeze-dried. The dry extract obtained (approximately $20 \mathrm{mg}$ ) was washed with ethanol, the excess of ethanol was removed by centrifugation ( $2220 \mathrm{~g}$ for $15 \mathrm{~min}$ at $20^{\circ} \mathrm{C}$ ) (Hasani et al. 2013), this step was repeated twice, then the material was put in DMAc for solvent exchange under magnetic stirring at room temperature overnight, the excess of DMAc was removed by centrifugation ( $2220 \mathrm{~g}$ for $15 \mathrm{~min}$ at $20^{\circ} \mathrm{C}$ ). After solvent exchange steps, 2 $\mathrm{mL}$ of DMAc/LiCl $9 \%(\mathrm{v} / \mathrm{w})$ were added to the vial containing the sample and allowed to stir magnetically at $4{ }^{\circ} \mathrm{C}$ for dissolution. 
The final concentration of the samples is $10 \mathrm{~g} / \mathrm{L}$. The dissolution was stopped by addition of pure DMAc. The final concentration of samples in DMAc/LiCl $0.9 \%(\mathrm{v} / \mathrm{w})$ is $1 \mathrm{~g} / \mathrm{L}$.

202 Before injection, the samples were filtered through a $0.45 \mu \mathrm{m}$ polytetrafluoroethylene

203 (PTFE).

Transmission Electron Microscopy (TEM). Droplets of CNCs suspensions at $0.8 \mathrm{~g} / \mathrm{L}$ were deposited on freshly glow-discharged carbon-coated microscope grids (200 mesh, Dalta Microscopies, France) for 2 min. The excess liquid was removed by filter paper, negatively stained with an aqueous solution of phosphotungstic acid at $10 \mathrm{~g} / \mathrm{L}$ for $2 \mathrm{~min}$ and dried just before TEM observation. We used a JEOL type transmission electron microscope (JEM-1230) operating at a voltage of $80 \mathrm{keV}$. The average dimensions (length and width) of the CNCs were determined from TEM images analysis of about 350 particles using the ImageJ software.

Atomic Force Microscopy (AFM). To determine the average thicknesses of the nanocrystals, the suspensions were diluted to $0.05 \mathrm{~g} / \mathrm{L}$ and then deposited on mica substrates. The measurements were carried out at room temperature by an Innova AFM (Bruker) using a monolithic silicon tip (TESPA, Bruker, spring constant $\mathrm{k}=42 \mathrm{~N} / \mathrm{m}$, frequency fO $=320 \mathrm{kHz}$ ). Image processing was performed with WSXM 5.0 software.

Small Angle Neutron Scattering (SANS) experiments. SANS experiments were carried out at room temperature using the small-angle PA20 and PAXY diffractometers at the Laboratoire Léon Brillouin (CEA/CNRS) in Saclay (France). Three configurations were used for PA20, covering a Q range from 0.0006 and $0.44 \AA-1(6 \AA$ at $1,1 \mathrm{~m}, 6 \AA$ at $8 \mathrm{~m}$, and $15 \AA$ at $17,5 \mathrm{~m})$, where $Q$ is the wave vector $(Q=4 \pi \sin \theta / 2$, where $\theta$ is the scattering angle and $\lambda$ is the neutron wavelength), and four configurations for PAXY, covering a Q range from 0.002 and $0.5 \AA-1$ ( $5 \AA$ at $1 \mathrm{~m}, 5 \AA$ at $3 \mathrm{~m}, 8,5 \AA$ at $5 \mathrm{~m}$ and $15 \AA$ at $6.7 \mathrm{~m}$ ). $\mathrm{CNCs}$ dispersions of $2 \mathrm{~g} / \mathrm{L}$ in $2 \mathrm{mM} \mathrm{NaCl}$ were prepared at $20^{\circ} \mathrm{C}$ then

228 extensively dialyzed against D2O to obtain the best possible contrast as well as to 229 reduce as much as possible the incoherent scattering then systematically freshly 230 sonicated for $10 \mathrm{~s}$ and loaded in quartz cells (Hellma) of small path length ( 1 and $2 \mathrm{~mm}$ ). 231 To determine the CNCs dimensions, the data were fitted with Sasview software. Several 232 fitting models were tried using the form factor of a parallelepiped with a rectangular 233 section, averaged over all space orientations, constituted a perfectly fitting model of the rodlike CNCs (Cherhal, Cousin, and Capron 2015). Aggregation experiments in solution were performed on suspensions at $2 \mathrm{~g} / \mathrm{L}$ of CNC-I and CNC-II in 2, 50 and $100 \mathrm{mM} \mathrm{NaCl}$. The suspensions were measured after sonication. 


\section{Results}

\subsection{Structural description}

The XRD patterns of cotton native, mercerized and hydrolyzed samples are shown in figure 1.
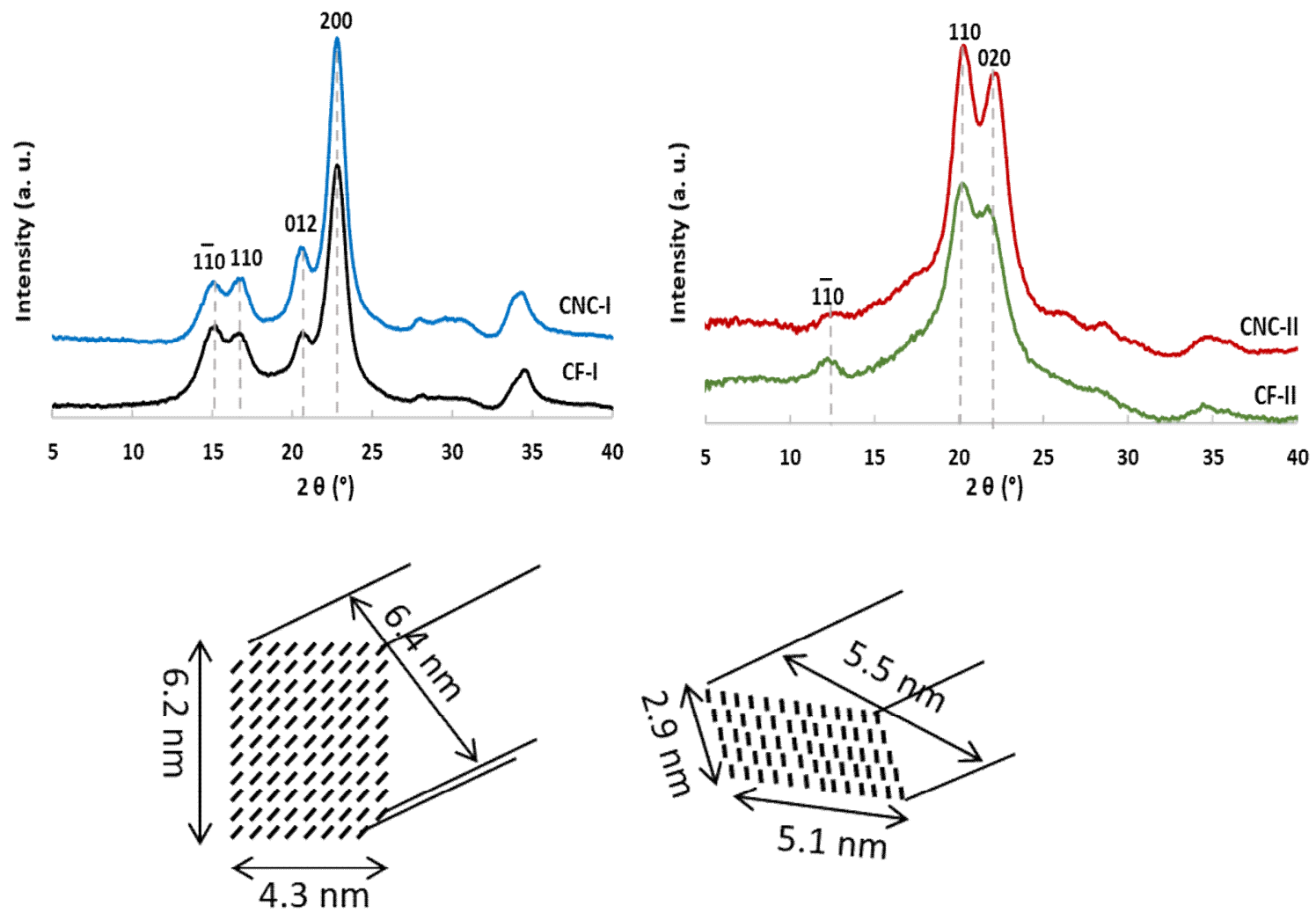

Figure 1. X-ray diffraction patterns of cotton fibers in native (CF-I) and mercerized CF-II forms and their respective hydrolysed cellulose nanocrystals in the native (CNC-I) and mercerized (CNC-II) forms and cross-sections of elementary crystallites deduced from the analysis of peak broadening (the indexation of corresponding lattice planes is described in Supporting Information).

The diffractions patterns of CF-I and CNC-I are typical of cellulose I with the presence of diffraction peaks at $15.1^{\circ}, 16.9^{\circ}, 20.7^{\circ}$ and $22.8^{\circ}$ corresponding to (1-10), (110), (012/102) and (200) crystallographic planes respectively. After mercerization, the crystallinity index ( $\mathrm{Cl}_{\mathrm{XRD}}$ ) of CF-II decreased. For the mercerized sample, CF-II and CNC-II at $12.3^{\circ}, 20.0^{\circ}$ and $21.7^{\circ}$ corresponding to the (1-10), (110) and (020) reflections respectively (Nishiyama, Kuga, and Okano 2000; Duchemin 2015; Isogai et al. 1989), but also traces of cellulose I residuals can be recognized at $15.1^{\circ}$ and $16.9^{\circ}$ (Figure 1 ). This allomorphic modification was achieved without loss in mass (Table 1). XRD peak analysis (See values in SI) allowed representation of the crystals (Fig. 1). The (1-10) and (110) crystalline planes count interplane dimension of $0.61 \mathrm{~nm}$ and $0.54 \mathrm{~nm}$ respectively (Sugiyama et al. 1991; Goussé et al. 2002). Similarly, for CNC-II, $0.72 \mathrm{~nm}$ and $0.44 \mathrm{~nm}$ distances are taken for (1-10) and (110) respectively (Kolpak, Weih, and Blackwell 1978; 
Table 1: Weight fraction (yield) recovered after treatment, Crystallinity index ( $\mathrm{Cl}$ ) calculated from XRD (CIXRD), Mean Cl calculated from solid-state NMR (13C CP-MAS) spectra (CINMR). And deconvolution of the $\mathrm{C} 4$ region of $13 \mathrm{C}$ CP-MAS specrta.

\begin{tabular}{|l|c|c|c|c|c|c|}
\hline \multirow{2}{*}{ Samples } & \multirow{2}{*}{$\begin{array}{c}\text { Yield } \\
(\%)\end{array}$} & $\begin{array}{c}\text { CIXRD } \\
(\%)\end{array}$ & $\begin{array}{c}\text { CINMR } \\
(\%)\end{array}$ & crystalline & $\begin{array}{c}\text { Deconvolution of the C4 region } \\
\text { intermediary } \\
\text { crystalline } \\
\text { domain }\end{array}$ & amorphous \\
\hline CF-I & - & 60 & $67 \%$ & $25 \%$ & $42 \%$ & $\begin{array}{c}26 \% \text { Acc }+7 \% \\
\text { inAcc }\end{array}$ \\
\hline CNC-I & 64 & 65 & $75 \%$ & $36 \%$ & $39 \%$ & $25 \%$ \\
\hline CF-II & 100 & 40 & $72 \%$ & $58 \%$ & $14 \%$ & $28 \%$ \\
\hline CNC-II & 40 & 70 & $85 \%$ & $74 \%$ & $11 \%$ & $15 \%$ \\
\hline
\end{tabular}

Figure 2 shows the 13C CP-MAS NMR spectra of CF-I and CF-II and confirm the mercerization process with the two peaks at 88.1 and $86.9 \mathrm{ppm}$ in the CF-II spectrum that are characteristics of type II cellulose (Ibbett, Domvoglou, and Fasching 2007; Newman and Davidson 2004). CF-I had a CINMR of 67\%, and this crystallinity increased after acid hydrolysis. For CF-II, this CINMR increased up to $72 \%$ after mercerization and of $89 \%$ after subsequent hydrolysis (CNC-II preparation).

Based on work of Larsson et al. 1999 on cellulose I, the signals in the 86-92 ppm region that refer to crystalline domains were further decomposed. This deconvolution analysis discriminates an "in-core" ordered region from a "paracrystalline" organisation described as having intermediate order between amorphous and crystalline cellulose (Zuckerstätter et al. 2013) (Fig. 2). According to this analysis, original CF-I would contain only $25 \%$ of pure crystalline domain, $42 \%$ of so-called paracrystalline domain, and $33 \%$ of an amorphous domain divided in $26 \%$ of accessible and $7 \%$ of inaccessible amorphous domains (Table 1).

After acid hydrolysis, an increase of the relative area of crystalline peaks at 86-92 ppm is observed and the CINMR increased in accordance with DRX results. However, the selective analysis of crystalline and para-crystalline structure shows that the paracrystalline organization is only slightly decreased. The increase in crystallinity between CF-I and CNC-I is then correlated to a loss of amorphous part, the paracrystalline domains being much less affected. According to the model proposed by Larsson and also used by Wickholm, para-crystalline domains are structures surrounding 
nanocrystals in the nanofibers and less accessible than amorphous domains. This simultaneous loss in amorphous domain in the $80-86 \mathrm{ppm}$ region is visible as only one peak. It describes the amorphous part attributed to the accessible amorphous domain, revealing that the hydrolysis led to independent nanocrystals removing the entire inaccessible amorphous domain.

After mercerization, a typical spectrum of cellulose-Il evidenced the allomorphic transition. However, the $13 \mathrm{C}$ NMR spectrum of CF-II shows a signal characteristic of crystalline $\mathrm{C} 6$ of cellulose I representing about $4 \%$ of the total $\mathrm{C} 6$ signal. This residual crystalline cellulose I-type conformation results from an ineffective penetration of $\mathrm{NaOH}$ in crystalline domains; they are potentially dispersed in a random way as proposed by Kim et al (Kim et al. 2006).

The mercerization process of the nanofibers results in a slight increase of CINMR from CF-I to CF-II (Table 1), that is contradictory with XRD results. Simultaneously, a slight decrease of amorphous contribution is noticed from $33 \%$ to $28 \%$, and only one peak is observed that refers to only one amorphous type domain. Compared to this, the so-called paracrystalline region that usually refers to structures surrounding cellulose-I nanocrystals, undergo a sharp decrease from $42 \%$ to $14 \%$. The origin and structure of such state is still not clear (Larsson et al. 1999; Bregado et al. 2019), except that it is an intermediate (in terms of mechanical properties, hydrogen bonding and chain ordering) between crystalline and amorphous cellulose. After mercerization, a peak is clearly visible at $85.5 \mathrm{ppm}$ (Fig. 2) refers to that imperfect crystalline region (or similarly to an ordered amorphous region). Such peak was previously observed and attributed to partially ordered cellulose (Ibbett, Domvoglou, and Fasching 2007). It results that only one type of the amorphous structure is remaining in slightly reduced amount, and that an important part of the paracrystalline-I structure that is presumably surrounding the crystalline domains formed by mercerization is lost.

Acid hydrolysis of the mercerized cellulose occurs with a loss of mass (yield 40\%) but without much changes of the peak attributed to intermediate structure. The same trend is then observed for both CNC-I and CNC-II. This was already reported by (Wickholm et al. 2001). It implies that acid hydrolysis removes amorphous regions contrary to mercerization process that strongly impact paracrystalline/intermediate domains. The same fraction of $4 \%$ of cellulose I observed in CF-II was recovered in the CNC-II sample. 

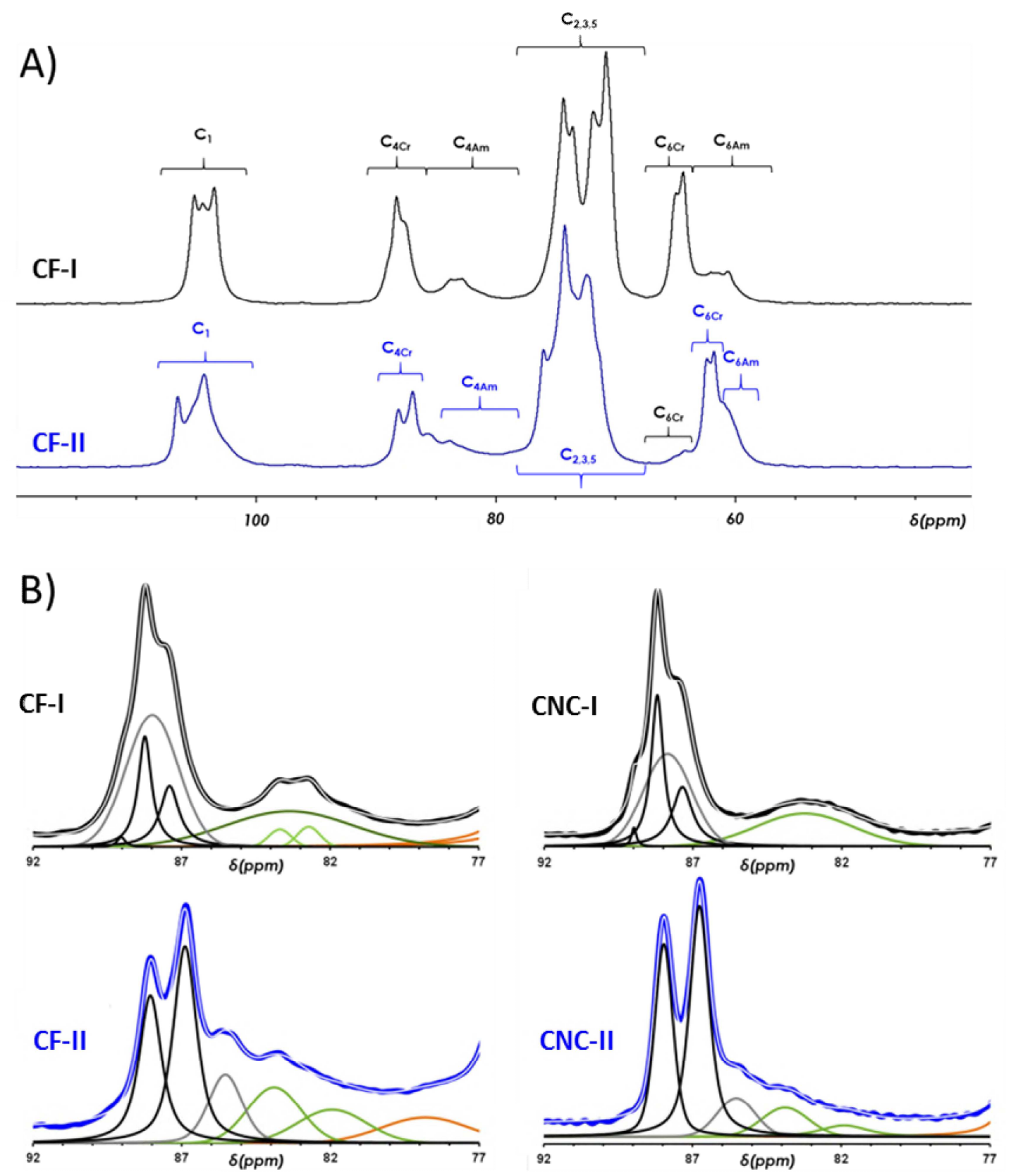

Figure 2. A) ${ }^{13} \mathrm{C}$ CP-MAS NMR spectra of CF-I and CF-II and B) deconvolution of C4 region of CF-I, CF-II, CNC-I and CNC-II NMR spectra with crystalline forms (black), paracrystalline (grey) and amorphous (green).

The results obtained by XRD and NMR are however controversial. The loss of crystallinity observed by XRD after mercerization is not observed by NMR (Table 1). In solid-state NMR, considering only the C4 region, chemical shifts are influenced by the conformation of carbon atoms in glycosidic chains, which may be involved in a crystalline, paracrystalline or amorphous structure. For XRD analysis, beyond crystallite orientation, it is directly the crystal lattice that is identified. It is therefore easy to imagine that parts of chains may have conformations related to those of crystal lattices without having a dimension that allows XRD to identify them as such, explaining a higher value of $\mathrm{Cl}$ by NMR. The variations observed can then be linked to the ability of each technic to detect imperfect organizations. NMR assumes that all the carbons involved are in a crystalline structure at short scale, analyzing crystalline and paracrystalline 
organizations in the so-called CINMR, and distinguishes these forms from the amorphous with signals shifted to lower ppm values. On the opposite XRD analysis requires longer scale organization, the presence of paracrystalline organizations being included in the widening peaks attributed to amorphous domains.

As a result, a major modification during mercerization comes from this intermediate state that is reformed in lower amount after swelling in $\mathrm{NaOH}$ and recrystallization process. Also mercerization leads to more crystalline domains that seem more discontinuous than the former. Such structures are not fully detected by XRD analysis but assumed by NMR as globally crystalline. Furthermore, only one amorphous peak is visible after mercerization by NMR implying only one type of amorphous area. This might reveal a more homogeneous but less organized system, with more imperfections, which is also in accordance with the increased susceptibility to acid hydrolysis of CF-II. After hydrolysis, imperfection is removed and highly crystalline particles are recovered as detected by both XRD and NMR analyses.

\subsection{Molar mass characterization}

In order to follow the process at a molecular level, the native and mercerized fibers have been dissolved in DMAC/LiCl and injected in a SEC-MALLS-DRI device. This experiment allowed determining the molar mass $(\mathrm{Mw})$ distribution of individual cellulosic chains. It may determine whether the process that involved $\mathrm{NaOH}$ at a high concentration had an impact on the glucosidic chains length. The fractionation mode using a size exclusion chromatographic process, the larger molecules came out first. Both fibers were found to have an average molar mass of $560000 \mathrm{~g} / \mathrm{mol}$ with a low polydispersity (table 2). Just a slight shift to higher retention volumes seemed to underline more flexibility of CF-I. However, it is here demonstrated that mercerization treatment of native cellulose fibers through $\mathrm{NaOH}$ swelling does not induce any molecular disruption.

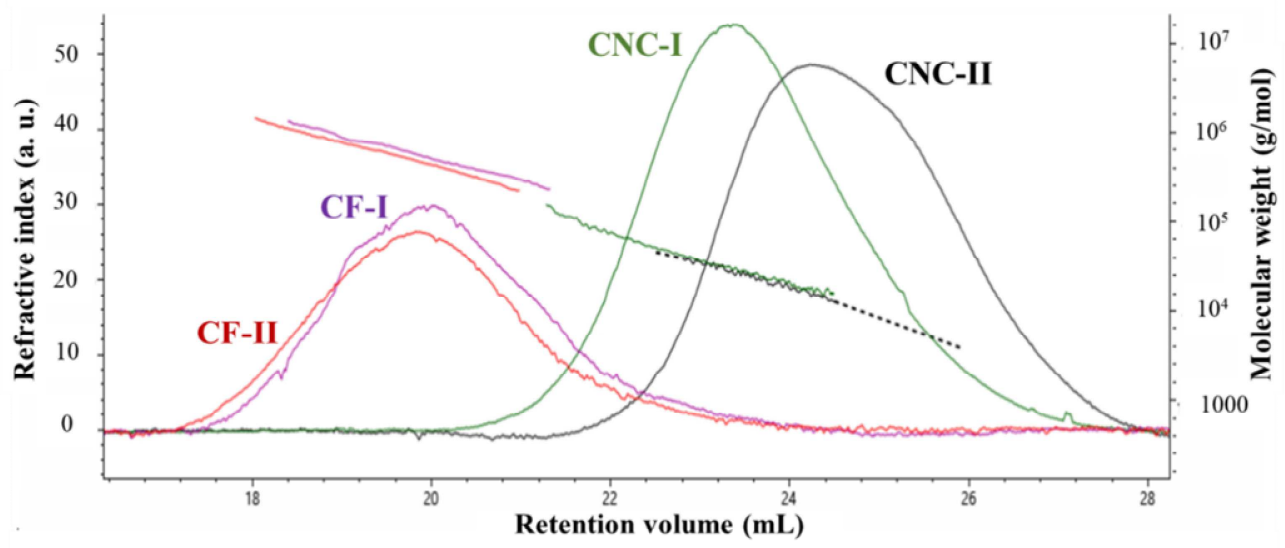

Figure 3. Dissolution profiles of samples obtained by SEC-MALLS-DRI. The two nanofibers (CF-I in purple and CF-II in red) are eluted at low retention volumes whereas the nanocrystals are eluted at higher elution volumes (CNC-I green and CNC-II black).

Table 2. Weight average molar masses $\left(\overline{\boldsymbol{M}}_{w}\right)$, polydispersity $\left(\overline{\boldsymbol{M}}_{w} / \overline{\boldsymbol{M}}_{\boldsymbol{n}}\right)$ and degree of polymerization (DP) of individual chains of cellulosic fibers (CF-I and CF-II) and cellulose nanocrystals (CNC-I and CNC-II) solubilized in DMAC/LiCl 0.9\%. 


\begin{tabular}{|c|c|c|c|c|}
\hline Samples & $\mathrm{M}_{\mathrm{w}}(\mathrm{g} / \mathrm{mol})$ & $\mathrm{M}_{\mathrm{w}} / \mathrm{M}_{\mathrm{n}}$ & $\mathrm{DP}_{\mathrm{w}}$ & $\mathrm{DP}_{\mathrm{n}}$ \\
\hline CF-I & $565000 \pm 47000$ & 1.3 & 3487 & 2683 \\
\hline CF-II & $556000 \pm 43000$ & 1.3 & 3432 & 2640 \\
\hline CNC-I & $41000 \pm 1000$ & 1.2 & 253 & 210 \\
\hline CNC-II & $22000 \pm 1000$ & 1.2 & 135 & 112 \\
\hline
\end{tabular}

388

Similarly, both CNCs have been solubilized in DMAc/LiCl $9 \%(v / w)$ for $\mathrm{Mw}$ distribution determination. They appear logically at larger retention volume compared to the fibers (Figure 3 ) indicating a significant decrease in the hydrodynamic volume of the chains. The acid hydrolysis of the fibers led to a clear decrease of the $\mathrm{Mw}$, from $560000 \mathrm{~g} / \mathrm{mol}$ for both fibers, down to $41000 \mathrm{~g} / \mathrm{mol}$ for CNC-I and to $22000 \mathrm{~g} / \mathrm{mol}$ for CNC-II (Table 2). Conversely to mercerization that did not affect the chain length, after the hydrolysis, the degree of polymerization (DP) of CNC-II is about half as low as CNC-I. Furthermore, the Mw distribution curves of CNC-II were shifted to lower retention volumes, but superimposed on a large domain illustrating the same proportion in occupied volume. In other words, CNC-II is similar in conformation but smaller.

Simultaneously, Mw distributions of the CNCs directly in suspension in water (without solubilization step) were obtained using A4F-MALLS-DRI analysis (Figure 4). The fractionation being carried out by a cross-flow device, the smaller molecules came out first. The shift to lower elution time for the CNC-II compared to CNC-I confirmed the lower hydrodynamic volumes of CNC-II. The Mw measured were also much lower (Table 3), with $36.106 \mathrm{~g} / \mathrm{mol}$ and $11.106 \mathrm{~g} / \mathrm{mol}$ for CNC-I and CNC-II respectively. These values are in agreement with the results found by SEC-MALLS-DRI device.

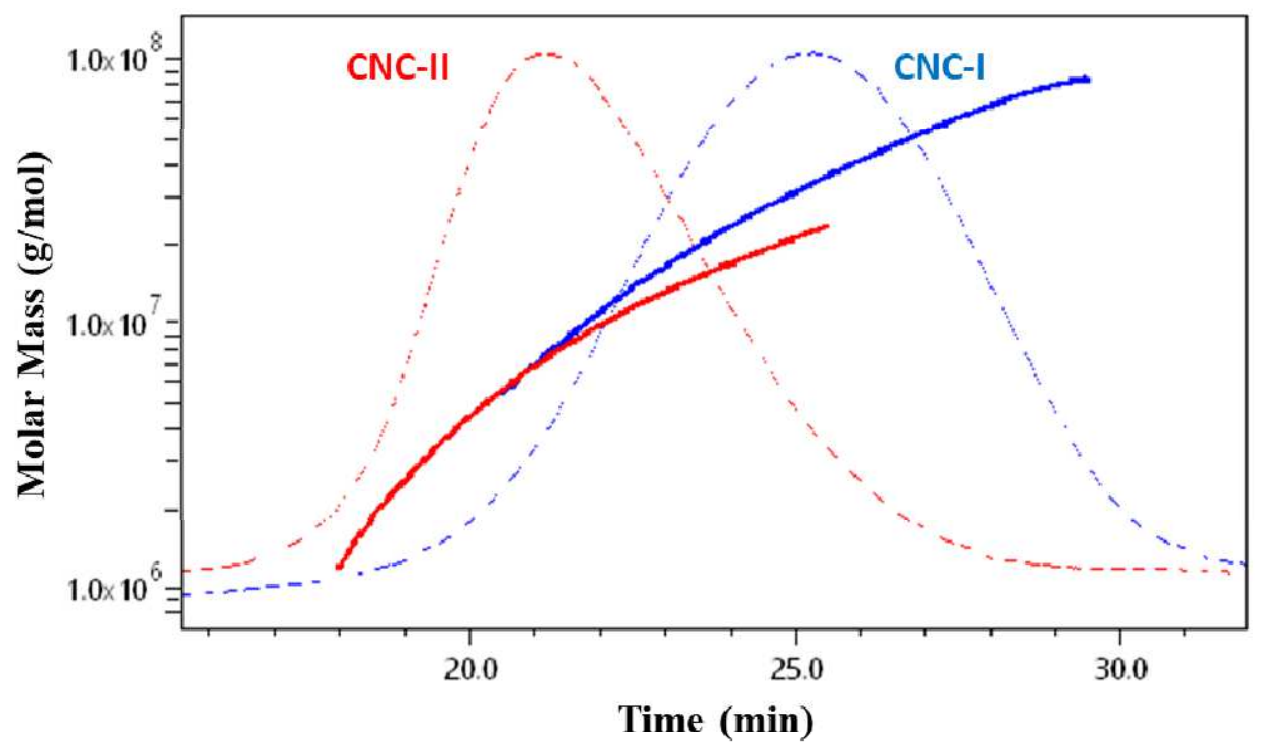

Figure 4. Distribution of molar masses of suspensions of CNC-I (blue) and CNC-II (red) in water, and RI signal (doted curves).

Dividing from both CNCs the molar mass obtained in crystalline form (Table 3) to that of their individual chains (Table 2), the packing appeared decreasing from 878 to 500 chains for CNC-I and CNC-II respectively. This is a very high value compared to the 
dimensions of the elementary CNC. This evidences that some aggregation is remaining. 416 However, it appears clearly that the mercerized CNCs are two to three times smaller in 417 length and packing. It results that the crystalline domains in NF-II are shorter with a DP 418 less than half of those in NF-I.

\subsection{Characterization of cellulose nanocrystals morphology}

The morphology of native and mercerized CNCs was characterized and compared by TEM, AFM and SANS. Figure 5 shows TEM and AFM images of native and mercerized CNCs. Both CNCs are in the form of rigid rods with shorter CNC-II. The average lengths of $118 \pm 65 \mathrm{~nm}$ and $65 \pm 22 \mathrm{~nm}$ were determined for CNC-I and CNC-II respectively (Table 3). This is in accordance with previous results (Neto et al. 2016). Selecting individual CNCs in order to measure elemental nanocrystals, CNC-I and CNC-II have the same individual width of $7 \pm 3 \mathrm{~nm}$. More surprisingly and differently from what was previously reported by (Neto et al. 2016), the average thicknesses found by AFM were $6.0 \pm 2.4 \mathrm{~nm}$ and $3.4 \pm 1.5 \mathrm{~nm}$ for CNC-I and CNC-II respectively (Table 3 ). The thickness reduced by half value is noticeable.
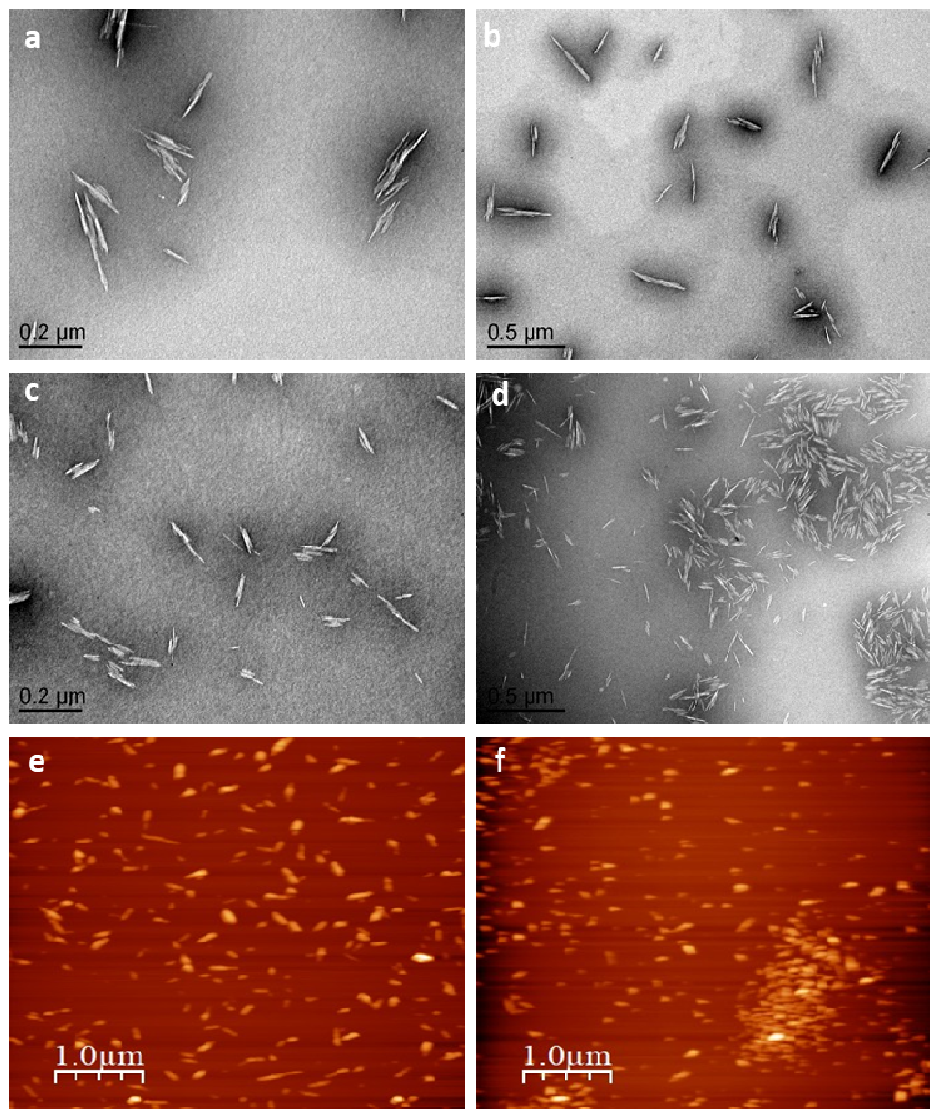

Figure 5. TEM images of CNC-I $(a, b)$ and CNC-II $(c, d)$ and AFM images of CNC-I (e) and CNC-II (f)

Table 3. Weight average molar masses ( $\mathrm{Mw}$ ), and polydispersity ( $\mathrm{Mw} / \mathrm{Mn}$ ) of CNC-I and CNC-II 


\begin{tabular}{|c|c|c|c|c|c|c|c|c|}
\hline Samples & \multirow{2}{*}{$\begin{array}{c}\overline{\mathrm{M}}_{\mathrm{w}} \\
\left(10^{6} \mathrm{~g} / \mathrm{mol}\right)\end{array}$} & \multirow{2}{*}{$\overline{\mathrm{M}}_{\mathrm{w}} / \overline{\mathrm{M}}_{\mathrm{n}}$} & \multicolumn{2}{|c|}{ Length(nm) } & \multicolumn{2}{c|}{ Width (nm) } & \multicolumn{2}{c|}{ Thickness (nm) } \\
\cline { 4 - 9 } & & & SANS & TEM & SANS & TEM & SANS & AFM \\
\hline CNC-I & $36 \pm 1$ & 1.5 & $175 \pm 25$ & $118 \pm 65$ & $21 \pm 1$ & $7 \pm 3$ & $6.5 \pm 0.5$ & $6.0 \pm$ \\
& & & & & & & & 2.5 \\
\hline CNC-II & $11 \pm 1$ & 1.5 & $75 \pm 25$ & $65 \pm 22$ & $22 \pm 2$ & $7 \pm 3$ & $3.5 \pm 0.5$ & $3.4 \pm 1.5$ \\
\hline 438 & & & & & & & & \\
\hline
\end{tabular}

The validation of these results was carried out in suspension of CNCs in water at 2 $\mathrm{mM} \mathrm{NaCl}$ by the fit of the curves obtained by small angle neutron scattering (SANS) 441 using the parallelepiped form factor (Figure 6). This measurement allows analysis in 442 dilute suspensions without drying step. CNC-I shows a higher intensity at low q revealing 443 a higher $\mathrm{Mw}$ and crosses the profile of CNC-II at intermediate Q. For both samples the 444 best fit obtained confirmed length and thickness values obtained by microscopy. Even if 445 some individual CNC must be present in suspension, a best fit is obtained for an average 446 width of $21 \mathrm{~nm}$ for both samples that corresponds to an average of 3 to 4 elementary 447 crystals associated laterally as already measured (Cherhal, Cathala, and Capron 2015; 448 Elazzouzi-Hafraoui et al. 2007)(Elazzouzi-Hafraoui, Nishiyama et al. 2008; Cherhal, 449 Cathala et al. 2015)(Elazzouzi-Hafraoui, Nishiyama et al. 2008; Cherhal, Cathala et al. 450 2015)(Elazzouzi-Hafraoui, Nishiyama et al. 2008; Cherhal, Cathala et al. 2015). The 451 lateral association is then not modified during the mercerization process. The 452 elementary cotton -based CNC-I is generally viewed with a squared cross-section, CNC-II 453 appears then with a rectangular cross-section. The values are in agreement with the 454 results found by A4F-MALLS-DRI and SEC-MALLS-DRI devices.

455

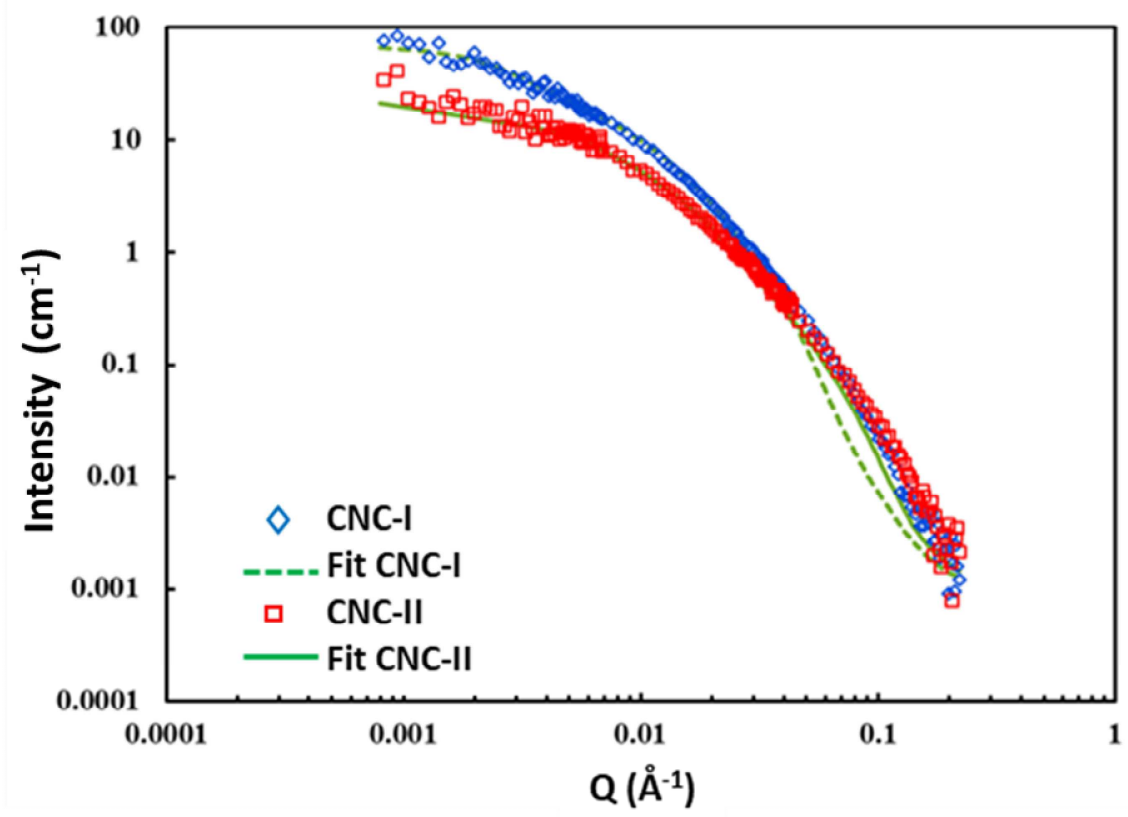

457 Figure 6. $I=f(Q)$ SANS curves of suspensions of CNC-I and CNC-II in water at $2 \mathrm{~g} / \mathrm{L}$ in NaCl $2 \mathrm{mM}$ 


\subsection{CNCs surface charge density}

The hydrolysis with sulfuric acid is known to graft anionic sulfate half esters (OSO3-) to the surface of the CNCs. These charges cause osmotic repulsion leading to a stable colloidal dispersion of CNCs in water. The same surface charge density is obtained for both CNCs as indicated by the sulfate content of $0.27 \%$ and the zeta-potential values of $-42 \mathrm{mV}$ for both CNC-I and CNC-II (Table 4). It implies a same susceptibility of both fiber surfaces to acid treatment.

Table 4. Sulphur content (S), surface charge density (SC) and zeta potential of CNC-I and CNC-II.

\begin{tabular}{|l|c|c|c|}
\hline Samples & $\mathrm{S}(\%)$ & $\mathrm{SC}(\mathrm{mmol} / \mathrm{g})$ & $\zeta$-potential $(\mathrm{mV})$ \\
\hline CNC-I & $0.278 \pm 0.09$ & $0.087 \pm 0.03$ & $-42.3 \pm 2.7$ \\
\hline CNC-II & $0.271 \pm 0.03$ & $0.085 \pm 0.01$ & $-41.9 \pm 1.9$ \\
\hline
\end{tabular}

\section{Discussion}

It results from this study that both nanocrystal types preserve trimer-like lateral association after mercerization while their thickness is divided by two after mercerization. They are shorter and more susceptible to hydrolysis, with larger interchain distances which is consistent with the lower density given for CNC-II. From our results, we can determine the average amount of chains per elementary crystal by several ways.

Based on the A4F-MALLS-RI results, both weight average (Mw) and number averaged $(\mathrm{Mn})$ molar mass are obtained. The number averaged molar mass of CNC is $24 \cdot 10^{6} \mathrm{~g} / \mathrm{mol}$ for CNC-I and $7 \cdot 10^{6} \mathrm{~g} / \mathrm{mol}$ for CNC-II (Table 5). By dividing these values by 3 , we obtain an average molar mass of $8 \cdot 10^{6} \mathrm{~g} / \mathrm{mol}$ for the elementary CNC-I and $2.4 \cdot 10^{6}$ $\mathrm{g} / \mathrm{mol}$ for the elementary CNC-II. These results with those obtained by SEC/MALLS allow determining the number of cellulosic chains in an elementary crystal. It gives 235 chains per elementary CNC-I and 133 chains per elementary CNC-II. This is large compared to theoretical calculations based on the crystals dimensions.

Considering the CNC completely crystalline and taking the crystal average dimensions obtained by microscopies, it leads to $7 \times 6.5 / 0.61 \times 0.54=162$ cellulose chains

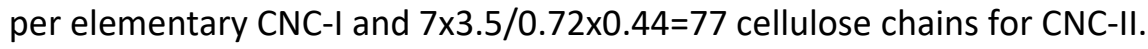

Calculating from the average crystalline dimensions from DRX analysis (see Fig 1), we obtain $4.3 \times 6.2 / 0.61 \times 0.54=80$ cellulose chains per elementary CNC-I and $2.9 \times 5.5 / 0.72 \times 0.44=50$ cellulose chains for CNC-II.

Whatever the calculation method (Table 5), about half of the former number of chains per elementary nanocrystal is recovered after mercerization. The chains are presumably mixed in the global fiber by interdigitation and during crystallization rearrange on shorter distances with smaller crystals packing less chains. However they seem more homogeneously distributed along the fiber.

This may indicate that during mercerization, all the chains of the fibril are redistributed forming a globally more regular fiber, but composed of smaller, more discontinuous and bi-oriented crystallites. A schematic model of the global organization is proposed in fig. 7. 
Table 5. Number averaged molar mass $\left(\bar{M}_{n}\right)$ of CNCs, elementary nanocrystal and individual chains and number of chains per individual CNC.

\begin{tabular}{|l|l|l|l|l|l|l|}
\hline CNCs & $\begin{array}{l}\overline{\mathrm{M}}_{\mathrm{n}} \\
\text { CNCs } \\
(\mathrm{g} / \mathrm{mol})\end{array}$ & $\begin{array}{l}\overline{\mathrm{M}}_{\mathrm{n}} \text { of } \\
\text { elementar } \\
\mathrm{y} \\
\text { nanocryst } \\
\mathrm{al}(\mathrm{g} / \mathrm{mol})\end{array}$ & $\begin{array}{l}\overline{\mathrm{M}}_{\mathrm{n}} \text { of } \\
\text { individual } \\
\text { chains } \\
(\mathrm{g} / \mathrm{mol})\end{array}$ & $\begin{array}{l}\text { Number of } \\
\text { chains/ } \\
\text { elementary } \\
\text { crystal } \\
\mathrm{Mn})\end{array}$ & $\begin{array}{l}\text { Number of } \\
\text { (from } \\
\text { elementary } \\
\text { crystal (from } \\
\text { microscopy) }\end{array}$ & $\begin{array}{l}\text { Number of } \\
\text { chains/ } \\
\text { elementary } \\
\text { crystal } \\
\text { (from XRD) }\end{array}$ \\
\hline CNC-I & $24 \pm 1 \cdot 10^{6}$ & $8 \cdot 10^{6}$ & $34000 \pm 1000$ & 235 & 162 & 82 \\
\hline CNC-II & $7 \pm 1 \cdot 10^{6}$ & $2.4 \cdot 10^{6}$ & $18000 \pm 1000$ & 133 & 77 & 50 \\
\hline
\end{tabular}

504

505

506

\section{Conclusions}

Using identical acid hydrolysis on native and mercerized NF, it is shown through a panel of techniques that the mercerization treatment doesn't degrade cellulosic chains ( $\mathrm{Mw}$ of $560000 \mathrm{~g} / \mathrm{mol}$ ) but limits the resistance to acid (yield of $64 \%$ and $40 \%$ for CNC-I and CNC-II respectively) and impacts the resulting CNCs. The thickness and length of nanocrystals are reduced preserving the lateral average association of three elementary crystals leading to molar masses of $40000 \mathrm{~g} / \mathrm{mol}$ and $11000 \mathrm{~g} / \mathrm{mol}$ for CNC-I and CNC-II respectively. Probing the internal structure showed more intermediary structure between ordered and amorphous. Also the two distinct (accessible / inaccessible) amorphous domains that are detected in cellulose-I are not detected in mercerized form, even before acid hydrolysis. This occurs with unchanged surface charge density but a reduction by half of the crystal thickness. Finally, mercerization has a major impact on crystal organization with a much lower chain packing per nanocrystal.

\section{Acknowledgement}


525 Authors are grateful to Nadege Beury for AFM images and Emilie Perrin for TEM images 526 with instruments from BIBS platform (INRAE, Nantes, France), and to Laboratoire Léon 527 Brillouin for providing neutron radiation facilities (CEA-Saclay Gif sur Yvette, France). 528 They are also grateful to Benoit Duchemin and Yoshiharu Nishiyama for very stimulating 529 discussions.

532 This project has received funding from Agence Nationale de la Recherche for the funding 533 of this work (project CELLOPLASM N ${ }^{\circ}$ ANR-16-CE07-0003-03), including the PhD grant of $\mathrm{SH}$. The authors are also grateful to INRAE for financial supports.

537 Ethics approval: No ethical approval required

540 References

541 Atalla, R. H., and David L. VanderHart. 1999. "The Role of Solid State 13C NMR Spectroscopy in 542 Studies of the Nature of Native Celluloses." Solid State Nuclear Magnetic Resonance 15 (1): 1-19.

543 Bregado, Jurgen Lange, Argimiro Resende Secchi, Frederico Wanderley Tavares, Dasciana de 544 Sousa Rodrigues, and Rossano Gambetta. 2019. “Amorphous Paracrystalline Structures from 545 Native Crystalline Cellulose: A Molecular Dynamics Protocol.” Fluid Phase Equilibria 491: 56-76.

546 Cherhal, Fanch, Bernard Cathala, and Isabelle Capron. 2015. "Surface Charge Density Variation to 547 Promote Structural Orientation of Cellulose Nanocrystals." Nordic Pulp \& Paper Research Journal 54830 (1): 126-31. https://doi.org/10.3183/npprj-2015-30-01-p126-131.

549 Cherhal, Fanch, Fabrice Cousin, and Isabelle Capron. 2015. "Influence of Charge Density and Ionic 550 Strength on the Aggregation Process of Cellulose Nanocrystals in Aqueous Suspension, as 551 Revealed by Small-Angle Neutron Scattering." Langmuir 31 (20): 5596-5602.

552 Duchemin, B. J. C. 2015. "Mercerisation of Cellulose in Aqueous $\mathrm{NaOH}$ at Low Concentrations." 553 Green Chemistry 17 (7): 3941-3947.

554 Dupont, Anne-Laurence, and Gabrielle Harrison. 2004. "Conformation and Dn/Dc Determination 555 of Cellulose in N, N-Dimethylacetamide Containing Lithium Chloride." Carbohydrate Polymers 58 556 (3): 233-243.

557 Elazzouzi-Hafraoui, Samira, Yoshiharu Nishiyama, Jean-Luc Putaux, Laurent Heux, Frédéric 558 Dubreuil, and Cyrille Rochas. 2007. "The Shape and Size Distribution of Crystalline Nanoparticles 559 Prepared by Acid Hydrolysis of Native Cellulose." Biomacromolecules 9 (1): 57-65.

560 Fink, Hans-Peter, and Burkart Philipp. 1985. "Models of Cellulose Physical Structure from the 561 Viewpoint of the Cellulose I $\rightarrow$ II Transition." Journal of Applied Polymer Science 30 (9): 37795623790.

563 French, Alfred D. 2014. "Idealized Powder Diffraction Patterns for Cellulose Polymorphs." 564 Cellulose $21(2): 885-896$. 
567 Goussé, Cécile, Henri Chanzy, Gérard Excoffier, Ludiwine Soubeyrand, and Etienne Fleury. 2002.

568 "Stable Suspensions of Partially Silylated Cellulose Whiskers Dispersed in Organic Solvents."

569 Polymer 43 (9): 2645-2651.

570 Habibi, Youssef, Lucian A. Lucia, and Orlando J. Rojas. 2010. "Cellulose Nanocrystals: Chemistry,

571 Self-Assembly, and Applications." Chemical Reviews 110 (6): 3479-3500.

572 Hasani, Merima, Ute Henniges, Alexander Idström, Lars Nordstierna, Gunnar Westman, Thomas 573 Rosenau, and Antje Potthast. 2013. "Nano-Cellulosic Materials: The Impact of Water on Their 574 Dissolution in DMAc/LiCl." Carbohydrate Polymers 98 (2): 1565-1572.

575 Ibbett, Roger N., Dimitra Domvoglou, and Mario Fasching. 2007. "Characterisation of the 576 Supramolecular Structure of Chemically and Physically Modified Regenerated Cellulosic Fibres by 577 Means of High-Resolution Carbon-13 Solid-State NMR." Polymer 48 (5): 1287-1296.

578 Isogai, Akira, Makoto Usuda, Takashi Kato, Toshiyuki Uryu, and Rajai H. Atalla. 1989. "Solid-State 579 CP/MAS Carbon-13 NMR Study of Cellulose Polymorphs." Macromolecules 22 (7): 3168-3172.

580 Kim, Nam-Hun, Tomoya Imai, Masahisa Wada, and Junji Sugiyama. 2006. "Molecular 581 Directionality in Cellulose Polymorphs." Biomacromolecules 7 (1): 274-280.

582 Kolpak, Francis J., Mark Weih, and John Blackwell. 1978. "Mercerization of Cellulose: 1. 583 Determination of the Structure of Mercerized Cotton." Polymer 19 (2): 123-131.

584 Kroon-Batenburg, L. M. J., B. Bouma, and J. Kroon. 1996. "Stability of Cellulose Structures Studied 585 by MD Simulations. Could Mercerized Cellulose II Be Parallel?" Macromolecules 29 (17): 56955865699.

587 Langan, P., Y. Nishiyama, and H. Chanzy. 1999. "A Revised Structure and Hydrogen-Bonding 588 System in Cellulose II from a Neutron Fiber Diffraction Analysis." Journal of the American 589 Chemical Society 121 (43): 9940-9946.

590 Langan, Paul, Yoshiharu Nishiyama, and Henri Chanzy. 2001. "X-Ray Structure of Mercerized 591 Cellulose II at 1 \AA Resolution." Biomacromolecules 2 (2): 410-416.

592 Larsson, Per Tomas, Eva-Lena Hult, Kristina Wickholm, Erik Pettersson, and Tommy Iversen. 1999. 593 "CP/MAS 13C-NMR Spectroscopy Applied to Structure and Interaction Studies on Cellulose I." 594 Solid State Nuclear Magnetic Resonance 15 (1): 31-40.

595 Li, Xia, Jun Li, Jie Gong, Yishan Kuang, Lihuan Mo, and Tao Song. 2018. "Cellulose Nanocrystals 596 (CNCs) with Different Crystalline Allomorph for Oil in Water Pickering Emulsions." Carbohydrate 597 Polymers 183: 303-310.

598 Medronho, Bruno, and Björn Lindman. 2015. "Brief Overview on Cellulose 599 Dissolution/Regeneration Interactions and Mechanisms." Advances in Colloid and Interface 600 Science 222 (August): 502-502-8. https://doi.org/10.1016/j.cis.2014.05.004.

601 Moon, R., A. Martini, J. Nairn, J. Simonsen, and J. Youngblood. 2011. Cellulose Nanomaterials 602 Review: Structure, Properties and Nanocomposites. Vol. 40. 603 http://explore.bl.uk/primo_library/libweb/action/display.do?tabs=detailsTab\&gathStatTab=true $604 \& c t=d i s p l a y \& f n=s e a r c h \& d o c=E T O C R N 293470090 \& i n d x=1 \&$ reclds=ETOCRN293470090. 
Neto, Wilson Pires Flauzino, Jean-Luc Putaux, Marcos Mariano, Yu Ogawa, Harumi Otaguro, Daniel Pasquini, and Alain Dufresne. 2016. "Comprehensive Morphological and Structural Investigation of Cellulose I and II Nanocrystals Prepared by Sulphuric Acid Hydrolysis." RSC Advances 6 (79): 76017-76027.

Newman, Roger H., and Tony C. Davidson. 2004. "Molecular Conformations at the CelluloseWater Interface." Cellulose 11 (1): 23-32. https://doi.org/10.1023/B:CELL.0000014778.49291.c6.

Nishiyama, Yoshiharu. 2009. "Structure and Properties of the Cellulose Microfibril." Journal of Wood Science 55 (4): 241-49. https://doi.org/10.1007/s10086-009-1029-1.

Nishiyama, Yoshiharu, Shigenori Kuga, and Takeshi Okano. 2000. "Mechanism of Mercerization Revealed by X-Ray Diffraction." Journal of Wood Science 46 (6): 452-457.

Nishiyama, Yoshiharu, Junji Sugiyama, Henri Chanzy, and Paul Langan. 2003. "Crystal Structure and Hydrogen Bonding System in Cellulose I $\alpha$ from Synchrotron X-Ray and Neutron Fiber Diffraction." Journal of the American Chemical Society 125 (47): 14300-306. https://doi.org/10.1021/ja037055w.

Okano, T., and A. Sarko. 1985. "Mercerization of Cellulose. II. Alkali-Cellulose Intermediates and a Possible Mercerization Mechanism." Journal of Applied Polymer Science 30 (1): 325-332.

Revol, J. F., A. Dietrich, and D. A. I. Goring. 1987. "Effect of Mercerization on the Crystallite Size and Crystallinity Index in Cellulose from Different Sources." Canadian Journal of Chemistry 65 (8): 1724-1725.

Revol, J.-F., H. Bradford, J. Giasson, R. H. Marchessault, and D. G. Gray. 1992. "Helicoidal SelfOrdering of Cellulose Microfibrils in Aqueous Suspension." International Journal of Biological Macromolecules 14 (3): 170-172.

Sèbe, Gilles, Frédérique Ham-Pichavant, Emmanuel Ibarboure, Akissi Lydie Chantal Koffi, and Philippe Tingaut. 2012. "Supramolecular Structure Characterization of Cellulose II Nanowhiskers Produced by Acid Hydrolysis of Cellulose I Substrates." Biomacromolecules 13 (2): 570-578.

Stipanovic, Arthur J., and Anatole Sarko. 1976. "Packing Analysis of Carbohydrates and Polysaccharides. 6. Molecular and Crystal Structure of Regenerated Cellulose II." Macromolecules 9 (5): 851-857.

Sugiyama, Junji, Roger Vuong, and Henri Chanzy. 1991. "Electron Diffraction Study on the Two Crystalline Phases Occurring in Native Cellulose from an Algal Cell Wall." Macromolecules 24 (14): 4168-4175.

Warwicker, J. O. 1967. "Effect of Chemical Reagents on the Fine Structure of Cellulose. Part IV. Action of Caustic Soda on the Fine Structure of Cotton and Ramie." Journal of Polymer Science Part A-1: Polymer Chemistry 5 (10): 2579-2593.

Wickholm, Kristina, Eva-Lena Hult, Per Tomas Larsson, Tommy Iversen, and Helena Lennholm. 2001. "Quantification of Cellulose Forms in Complex Cellulose Materials: A Chemometric Model." Cellulose 8 (2): 139-148.

Yanagisawa, Masahiro, and Akira Isogai. 2005. "SEC- MALS- QELS Study on the Molecular Conformation of Cellulose in LiCl/Amide Solutions." Biomacromolecules 6 (3): 1258-1265.

Zuckerstätter, Gerhard, Nicoleta Terinte, Herbert Sixta, and Kurt Christian Schuster. 2013. "Novel Insight into Cellulose Supramolecular Structure through 13C CP-MAS NMR Spectroscopy and 


\section{Figure captions:}

650 Figure 1. X-ray diffraction patterns of cotton fibers in native (CF-I) and mercerized CF-II forms and 651 their respective hydrolysed cellulose nanocrystals in the native (CNC-I) and mercerized (CNC-II) 652 forms.

Figure 2. A) ${ }^{13}$ C CP-MAS NMR spectra of CF-I and CF-II and B) deconvolution of C4 region of CF-I, CF-II, CNC-I and CNC-II NMR spectra with crystalline forms (black), paracrystalline (grey) and amorphous (green).

Figure 3. Dissolution profiles of samples obtained by SEC-MALLS-DRI. The two nanofibers (CF-I in purple and CF-II in red) are eluted at low retention volumes whereas the nanocrystals are eluted at higher elution volumes (CNC-I green and CNC-II black).

Figure 4. Distribution of molar masses of suspensions of CNC-I (blue) and CNC-II (red) in water, and RI signal (doted curves).

Figure 5. TEM images of CNC-I (a,b) and CNC-II (c,d) and AFM images of CNC-I (e) and CNC-II (f)

Figure 6. I = $f(Q)$ SANS curves of suspensions of CNC-I and CNC-II in water at $2 \mathrm{~g} / \mathrm{L}$ in $\mathrm{NaCl} 2 \mathrm{mM}$

Figure 7: Cross-sections of elementary crystallites deduced from the analysis of peak broadening in WAXS profiles from films of cotton (the indexation of corresponding lattice planes is described in Supporting Information), and schematic representation of a typical NF-I and NF-II deduced from the previous analyses.

\section{Table Captions:}

Table 1: Weight fraction (yield) recovered after treatment, Crystallinity index (Cl) calculated from XRD (CIXRD), Mean Cl calculated from solid-state NMR (13C CP-MAS) spectra (CINMR). And deconvolution of the $\mathrm{C} 4$ region of $13 \mathrm{C}$ CP-MAS specrta.

Table 2. Weight average molar masses $\left(\overline{\boldsymbol{M}}_{w}\right)$, polydispersity $\left(\overline{\boldsymbol{M}}_{w} / \overline{\boldsymbol{M}}_{\boldsymbol{n}}\right)$ and degree of polymerization (DP) of individual chains of cellulosic fibers (CF-I and CF-II) and cellulose nanocrystals (CNC-I and CNC-II) solubilized in DMAC/LiCl $0.9 \%$.

Table 3. Weight average molar masses (Mw), and polydispersity (Mw/Mn) of CNC-I and CNC-II dispersed in water determined by A4F-MALLS-DRI; and average dimensions determined from the SANS curve, TEM images and AFM images.

Table 4. Sulphur content (S), surface charge density (SC) and zeta potential of CNC-I and CNC-II.

Table 5. Number averaged molar mass $\left(\bar{M}_{n}\right)$ of CNCs, elementary nanocrystal and individual chains and number of chains per individual CNC. 
684

685

686

687

688

689

690

691

692

693

694

695

696

697

698

699

700

701

702

703

704

705

706

707

708

709

710
Cherhal, F., B. Cathala, et al. (2015). "Surface charge density variation to promote structural orientation of cellulose nanocrystals." Nordic Pulp and Paper Research Journal 30(2): 126-131.

Elazzouzi-Hafraoui, S., Y. Nishiyama, et al. (2008). "The shape and size distribution of crystalline nanoparticles prepared by acid hydrolysis of native cellulose." Biomacromolecules 9(1): 57-65.

Heise, K., G. Delepierre, et al. (2021). "Chemical Modification of Reducing End-Groups in Cellulose Nanocrystals." Angewandte Chemie-International Edition 60(1): 66-87.

Langan, P., Y. Nishiyama, et al. (1999). "A revised structure and hydrogen-bonding system in cellulose II from a neutron fiber diffraction analysis. "Journal of the American Chemical Society 121(43): 9940-9946.

Langan, P., Y. Nishiyama, et al. (2001). "X-ray structure of mercerized cellulose II at 1 angstrom resolution." Biomacromolecules 2(2): 410-416.

Neto, W. P. F., J. L. Putaux, et al. (2016). "Comprehensive morphological and structural investigation of cellulose I and II nanocrystals prepared by sulphuric acid hydrolysis. " RsC Advances 6(79): 76017-76027.

Nishiyama, Y. (2009). "Structure and properties of the cellulose microfibril." Journal of Wood Science 55(4): 241-249.

Okano, T. and A. Sarko (1985). "Mercerization of cellulose. 2 Alkali cellulose intermediates and a possible mercerization mechanism." Journal of Applied Polymer Science 30(1): 325-332.

Revol, J. F., A. Dietrich, et al. (1987). "Effect of mercerization on the crystallite size and crystallinity index in cellulose from different sources. " Canadian Journal of Chemistry-Revue Canadienne De Chimie 65(8): 1724-1725.

Tao, H., N. Lavoine, et al. (2020). "Reducing end modification on cellulose nanocrystals: strategy, characterization, applications and challenges." Nanoscale Horizons 5(4): 607-627. 
Figures
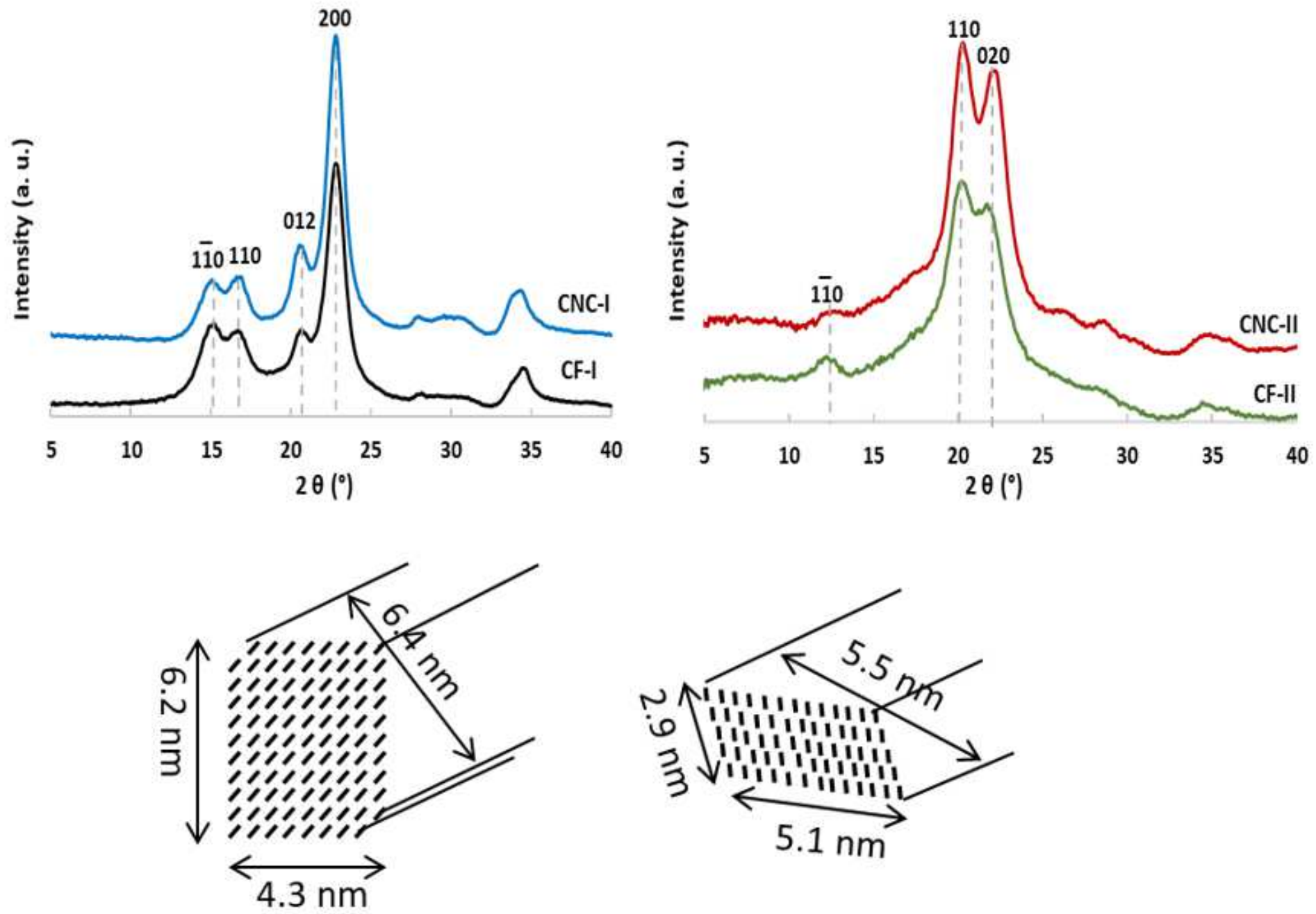

Figure 1

X-ray diffraction patterns of cotton fibers in native (CF-I) and mercerized CF-II forms and their respective hydrolysed cellulose nanocrystals in the native (CNC-I) and mercerized (CNC-II) forms. 

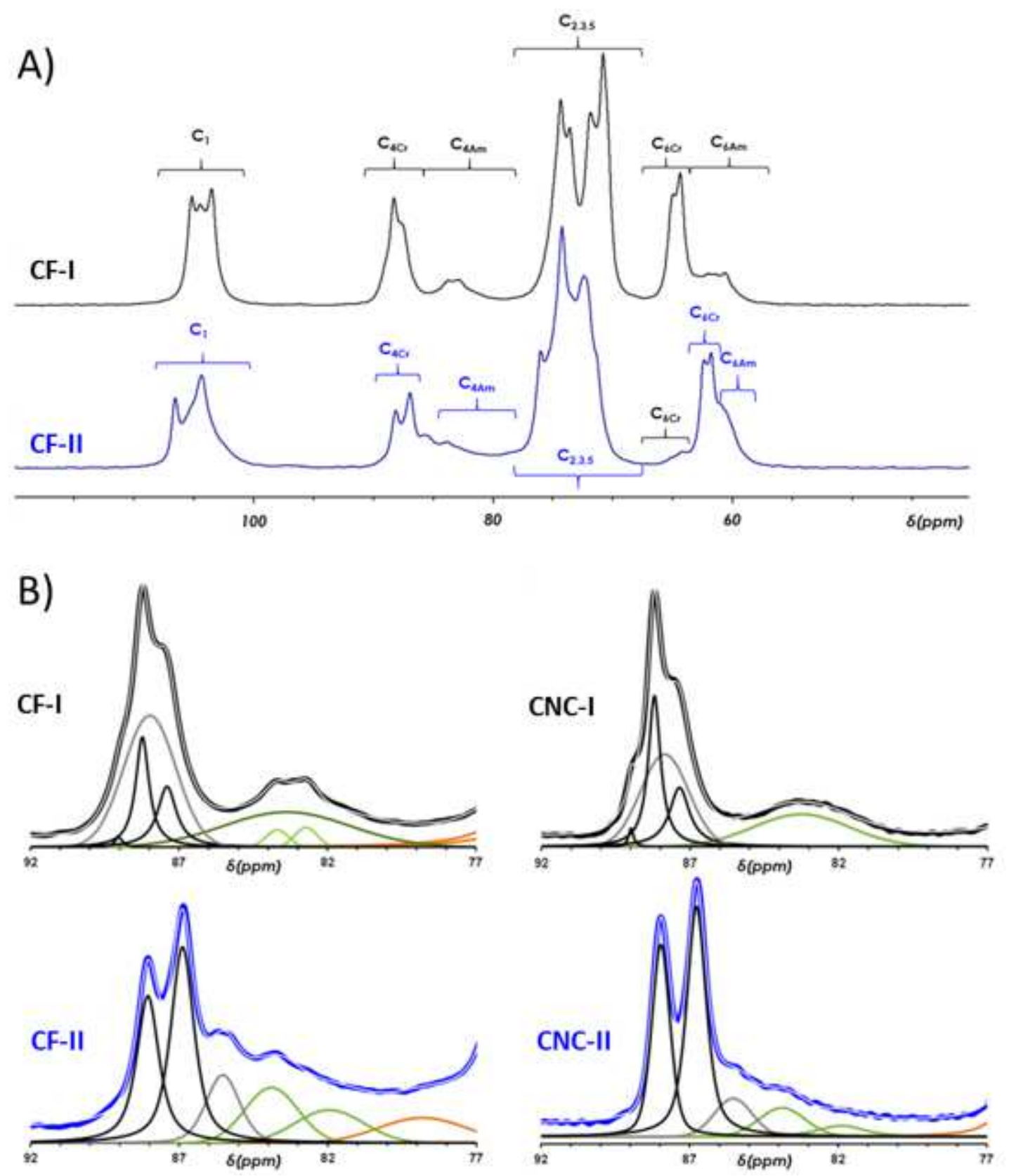

Figure 2

A) 13C CP-MAS NMR spectra of CF-I and CF-II and B) deconvolution of C4 region of CF-I, CF-II, CNC-I and CNC-II NMR spectra with crystalline forms (black), paracrystalline (grey) and amorphous (green). 


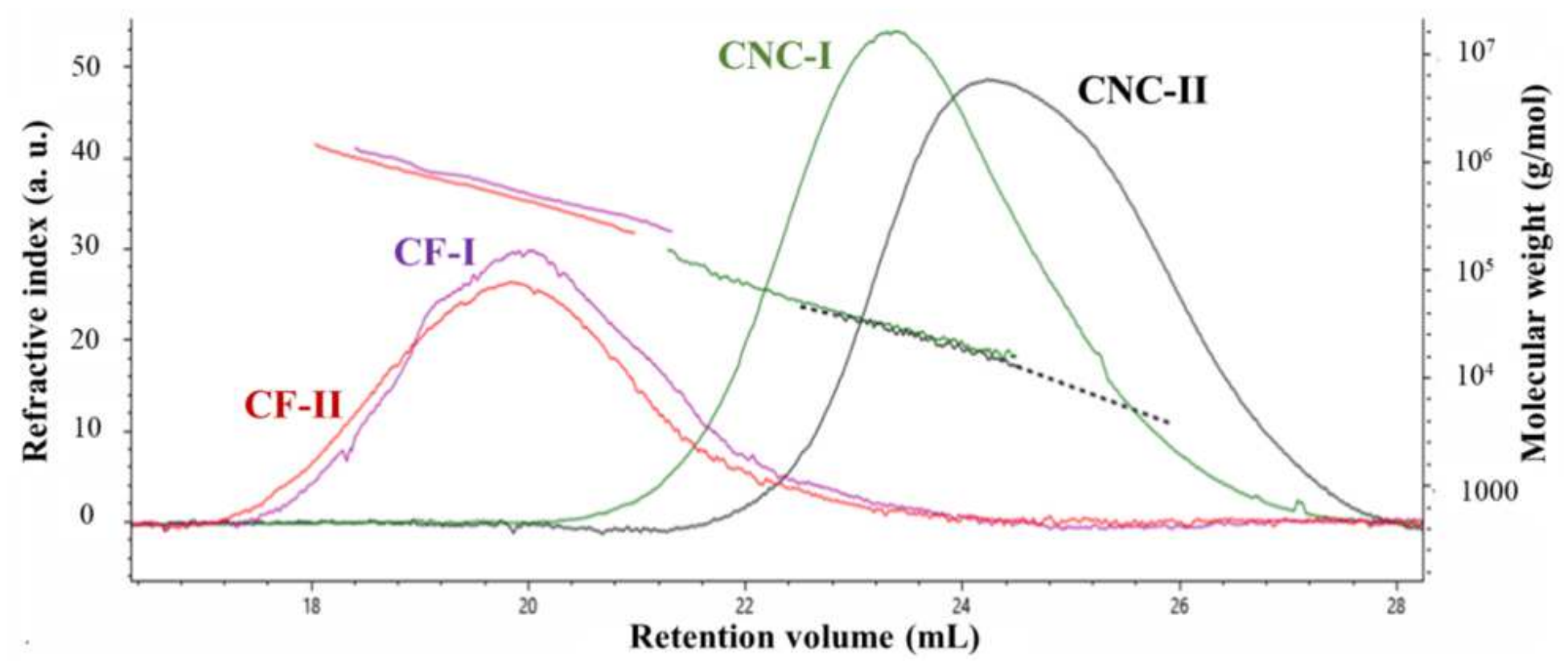

Figure 3

Dissolution profiles of samples obtained by SEC-MALLS-DRI. The two nanofibers (CF-I in purple and CF-II in red) are eluted at low retention volumes whereas the nanocrystals are eluted at higher elution volumes (CNC-I green and CNC-II black).

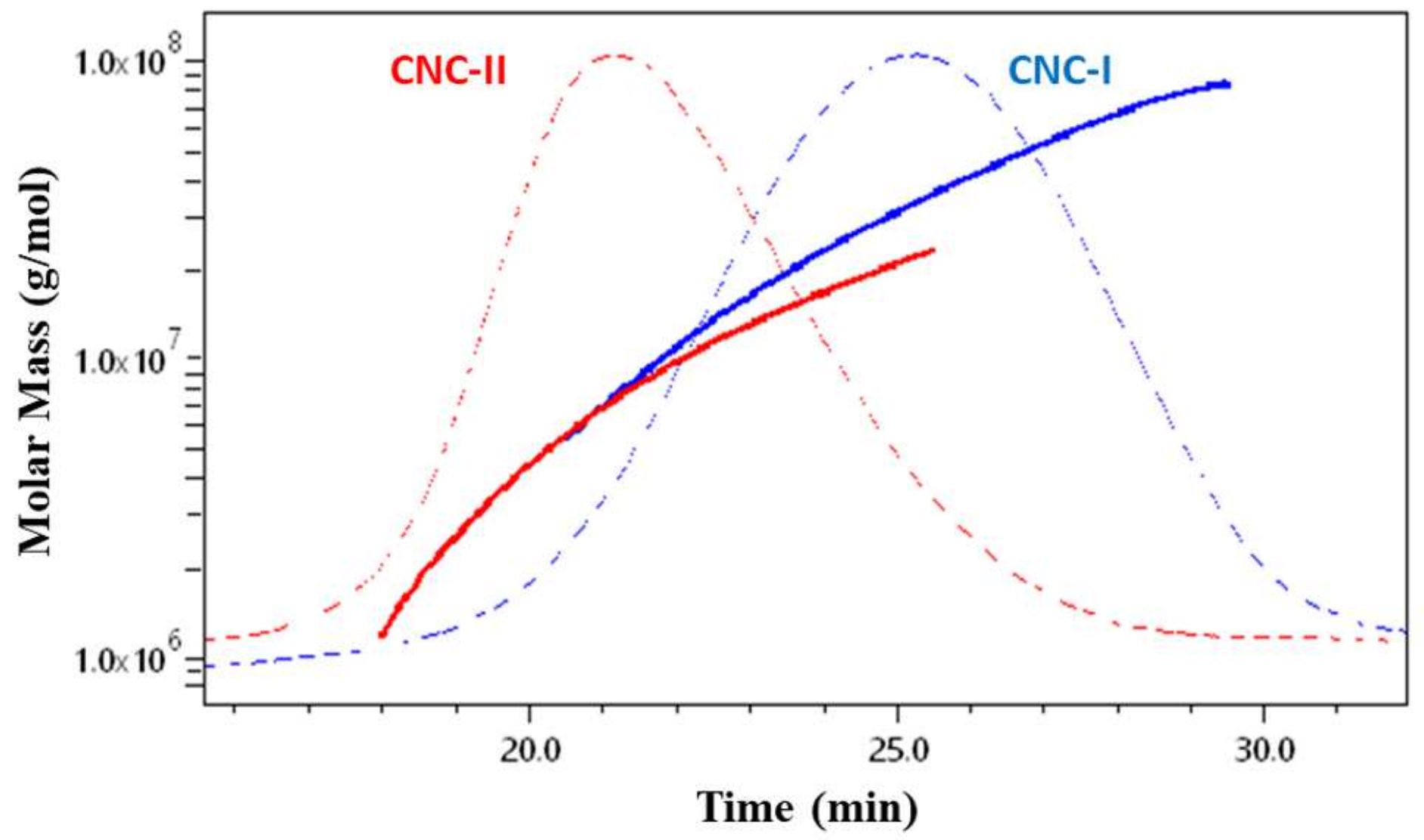


Figure 4

Distribution of molar masses of suspensions of CNC-I (blue) and CNC-II (red) in water, and RI signal (doted curves).
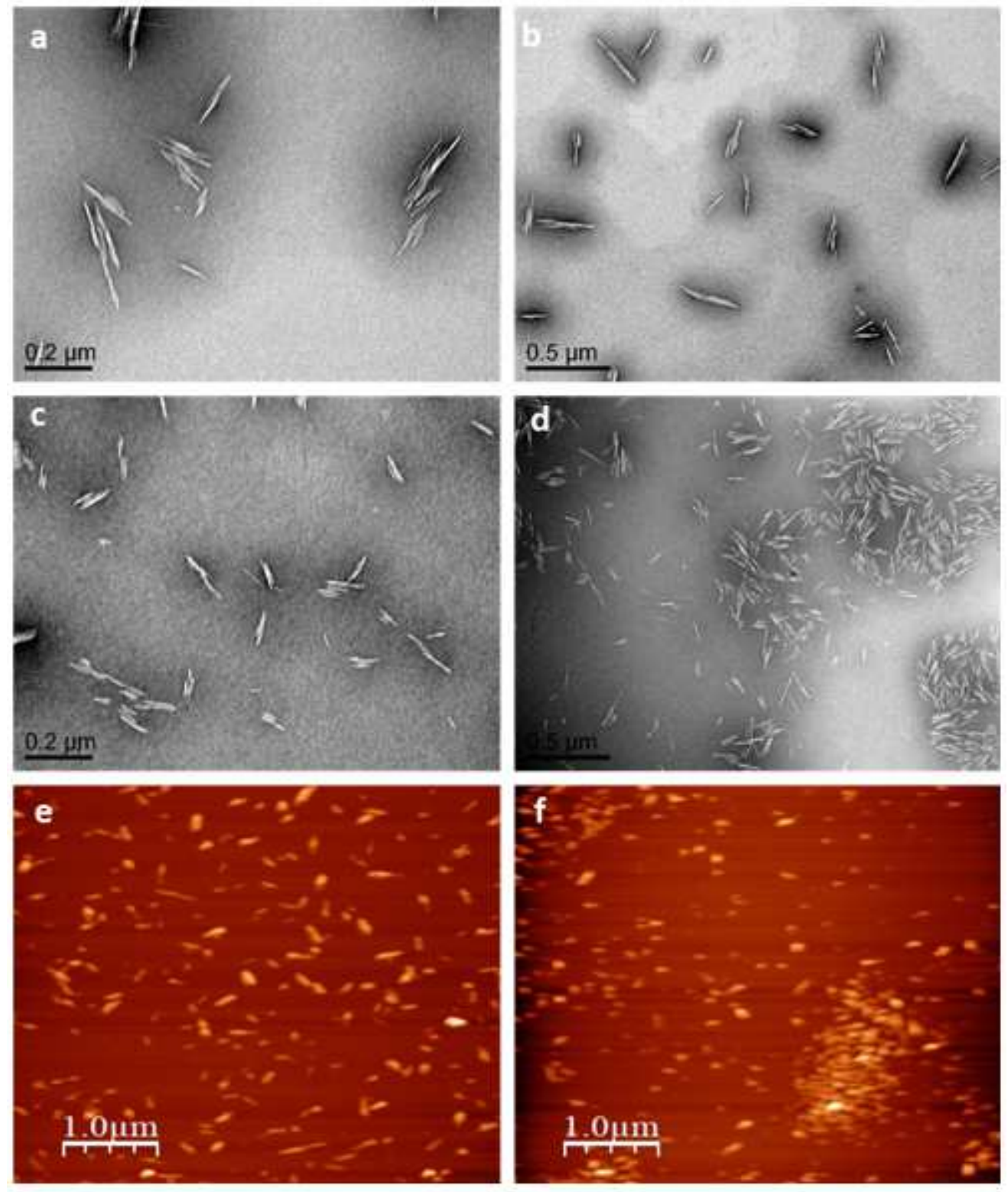

Figure 5

TEM images of CNC-I (a,b) and CNC-II (c,d) and AFM images of CNC-I (e) and CNC-II (f) 


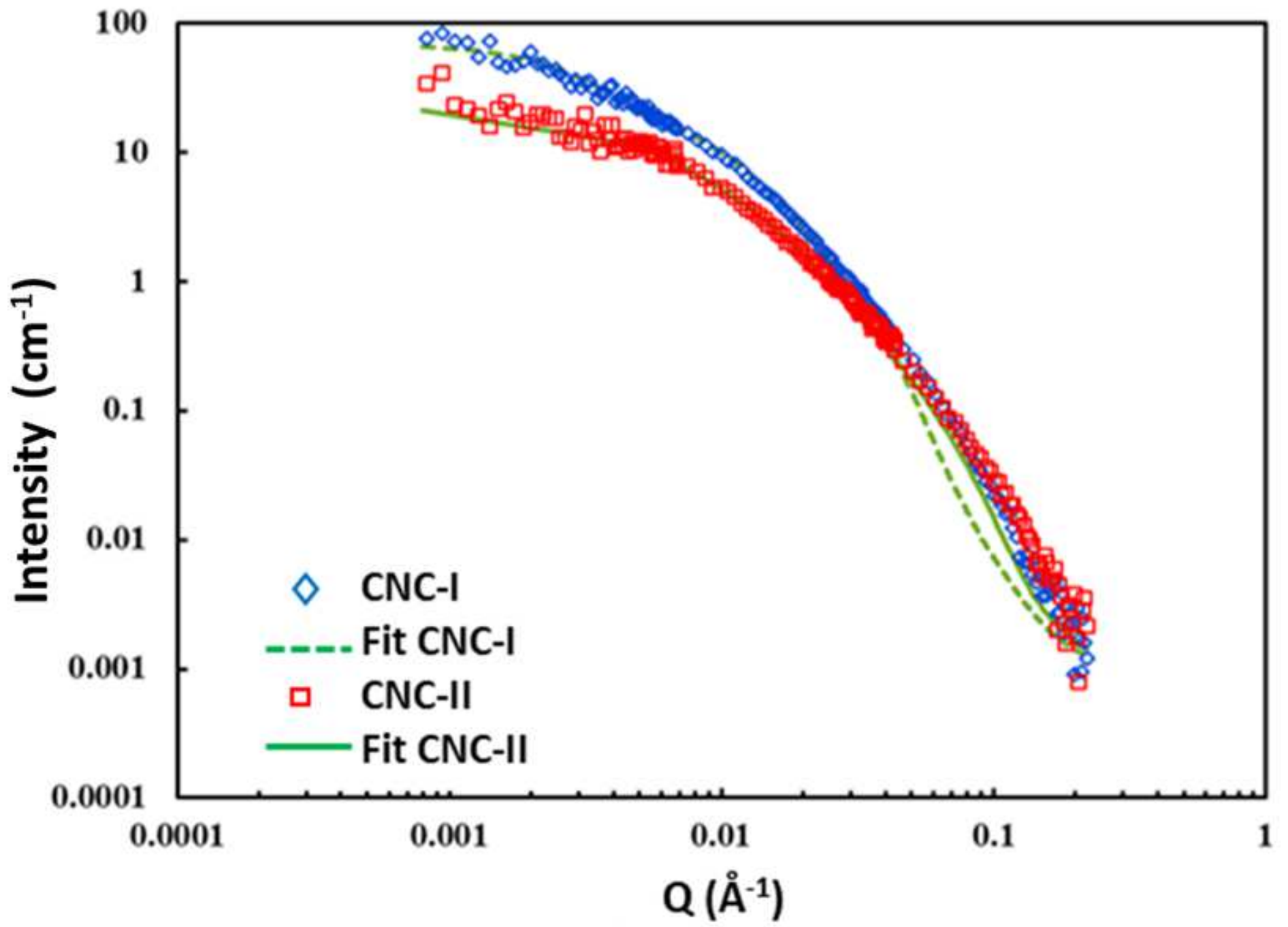

Figure 6

$\mathrm{I}=\mathrm{f}(\mathrm{Q})$ SANS curves of suspensions of CNC-I and CNC-II in water at $2 \mathrm{~g} / \mathrm{L}$ in $\mathrm{NaCl} 2 \mathrm{mM}$ 
CF-I

\section{crystalline \\ paracrystalline}

amorphous accessible + non acc

CF-II

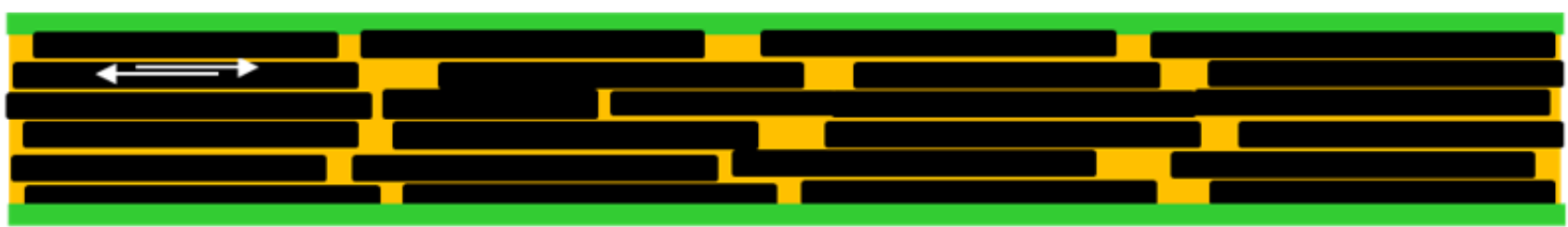

crystalline

Intermediary

amorphous

Figure 7

Cross-sections of elementary crystallites deduced from the analysis of peak broadening in WAXS profiles from films of cotton (the indexation of corresponding lattice planes is described in Supporting Information), and schematic representation of a typical NF-I and NF-II deduced from the previous analyses.

\section{Supplementary Files}

This is a list of supplementary files associated with this preprint. Click to download.

- SupplementaryInformation.docx 